LAWRENCE LIVERMORE N A T IO N A L LABORATORY

Recent Flash X-Ray Injector Modeling

T.L. Houck, D.T. Blackfield, J. Burke, Y-J Chen, J. Javedani, A. C. Paul

December 14, 2004 
This document was prepared as an account of work sponsored by an agency of the United States Government. Neither the United States Government nor the University of California nor any of their employees, makes any warranty, express or implied, or assumes any legal liability or responsibility for the accuracy, completeness, or usefulness of any information, apparatus, product, or process disclosed, or represents that its use would not infringe privately owned rights. Reference herein to any specific commercial product, process, or service by trade name, trademark, manufacturer, or otherwise, does not necessarily constitute or imply its endorsement, recommendation, or favoring by the United States Government or the University of California. The views and opinions of authors expressed herein do not necessarily state or reflect those of the United States Government or the University of California, and shall not be used for advertising or product endorsement purposes.

This work was performed under the auspices of the U.S. Department of Energy by University of California, Lawrence Livermore National Laboratory under Contract W-7405-Eng-48. 


\title{
Recent Flash X-Ray Injector Modeling
}

\author{
T.L. Houck, D.T. Blackfield, J. Burke, Y-J Chen, J. Javedani, and A.C. Paul
}

4 November 2004

\begin{abstract}
The injector of the Flash X-Ray (FXR) accelerator has a significantly larger than expected beam emittance. A computer modeling effort involving three different injector design codes was undertaken to characterize the FXR injector and determine the cause of the large emittance. There were some variations between the codes, but in general the simulations were consistent and pointed towards a much smaller normalized, rms emittance $(36 \mathrm{~cm}-\mathrm{mr})$ than what was measured $(193 \mathrm{~cm}-\mathrm{mr})$ at the exit of the injector using a pepperpot technique. The simulations also indicated that the present diode design was robust with respect to perturbations to the nominal design. Easily detected mechanical alignment/position errors and magnet errors did not lead to appreciable increase in the simulated emittance. The physics of electron emission was not modeled by any of the codes and could be the source of increased emittance. The nominal simulation assumed uniform Child-Langmuir Law emission from the velvet cathode and no shroud emission. Simulations that looked at extreme non-uniform cathode and shroud emission scenarios resulted in doubling of the emittance. An alternative approach was to question the pepperpot measurement. Simulations of the measurement showed that the pepperpot aperture foil could double the emittance with respect to the non-disturbed beam. This leads to a diplomatic explanation of the discrepancy between predicted and measured emittance where the fault is shared. The measured value is too high due to the effect of the diagnostic on the beam and the simulations are too low because of unaccounted cathode and/or shroud emission physics.
\end{abstract}

Fortunately there is a relatively simple experiment that can resolve the emittance discrepancy. If the large measured emittance value is correct, the beam envelope is emittance dominated at modest values of focusing field and beam radius. Measurements of the beam envelope on an imaging foil at the exit of the injector would lead to an accurate value of the emittance. If the emittance was approximately half of the measured value, the beam envelope is slightly space charge dominated, but envelope measurements would set reasonable bounds on the emittance value. For an emittance much less than $100 \mathrm{~cm}-\mathrm{mr}$, the envelope measurements would be insensitive to emittance. The outcome of this envelope experiment determines if a redesigned diode is needed or if more sophisticated emittance measurements should be pursued.

This work was performed Under the auspices of the U.S. Department of Energy by University of California Lawrence Livermore National Laboratory under contract No. W-7405-Eng-48 


\section{Table of Contents}

Table of Tables

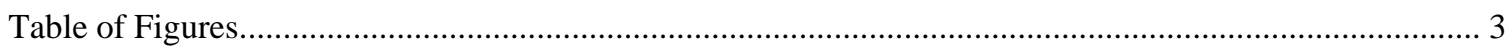

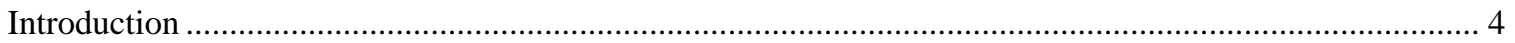

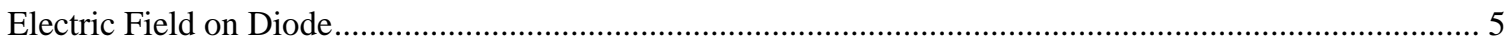

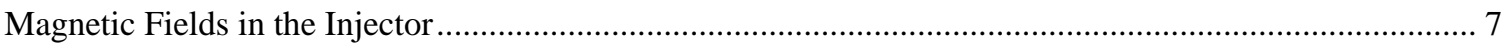

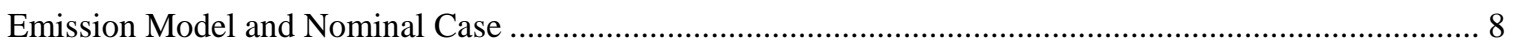

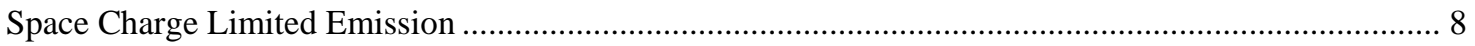

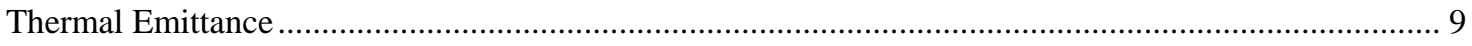

Time Dependent Emission and Transport ........................................................................................... 10

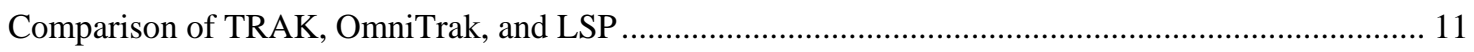

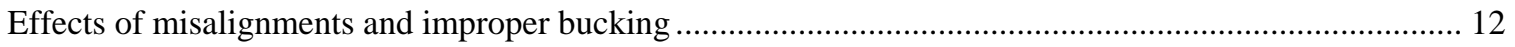

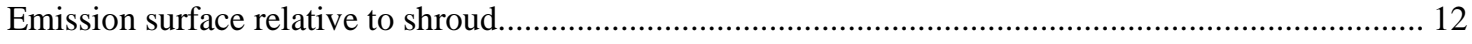

Variation in A-K gap............................................................................................................... 13

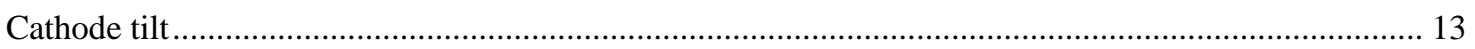

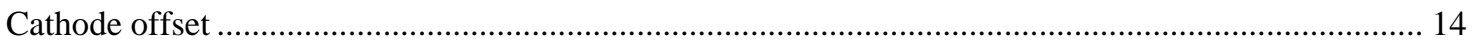

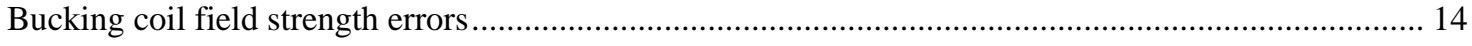

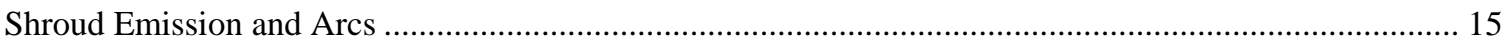

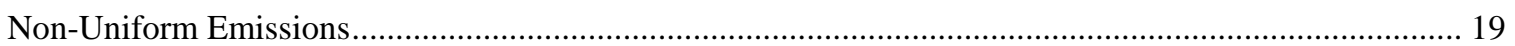

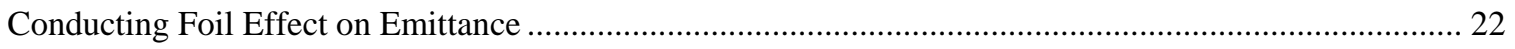

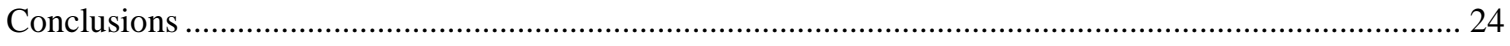

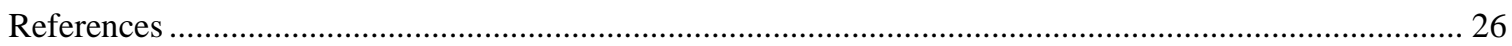

\section{Table of Tables}

Table 1. Measured emittance for high current linear induction accelerators.................................................... 4

Table 2. Emission current calculated by the various beam codes with the mesh symmetries used................ 8

Table 3. Effect of Alignment and Bucking Coil errors on Emittance.......................................................... 12

Table 4. Effect of increasing emittance on beam radius for different focusing field strengths. .................... 26 


\section{Table of Figures}

Figure 1. Photograph of the diode region and simulated geometry with equipotential line.

Figure 2. The longitudinal electric field plotted as a function of radial position at different axial positions. 6

Figure 3. The radial electric field plotted as a function of radial position at different axial positions. .......... 7

Figure 4. Layout of the injector solenoids with constant $\mathrm{A}_{\mathrm{z}}$ contours. ............................................. 8

Figure 5. The magnetic field is plotted as a function of longitudinal position for different radial distances.. 8

Figure 6. TRAK simulated electron orbits for the FXR injector. .................................................. 9

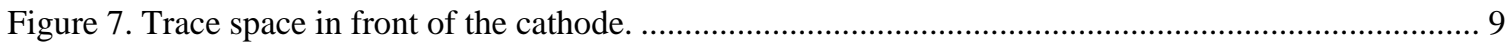

Figure 8. Emittance is shown as a function of axial position for three different angular spreads. .............. 10

Figure 9. Snapshot of the beam at five different times into the pulse: ................................................. 11

Figure 10. Comparison of the calculated rms radius between TRAK, OmniTrak, and LSP..................... 11

Figure 11. Comparison of the calculated emittance between TRAK, OmniTrak, and LSP. ..................... 11

Figure 12. Emittance is shown as a function of axial position for different cathode/shroud spacing.......... 12

Figure 13. Emittance is shown as a function of axial position for different A-K spacing........................ 13

Figure 14. Emittance is shown for different amounts of cathode tilt with respect to the axis. .................... 13

Figure 15. Emittance for different transverse offsets of the cathode from the z-axis............................ 14

Figure 16. Emittance as a function of axial position for different Bucking Coil currents. ........................ 15

Figure 17. Simulated electron orbits are shown emitted from both the cathode and shroud. ..................... 15

Figure 18. Maximum and rms radii for orbits from both the cathode and cathode-shroud are shown......... 16

Figure 19. Emittance for emission from both the cathode and cathode-shroud are shown. ...................... 16

Figure 20. Images of the FXR cathode showing a possible shroud arc. ............................................... 17

Figure 21. Three-dimensional depiction of emission from a small plasma bubble. ............................... 17

Figure 22. Comparison of emittance for the primary beam with and without emission from the shroud. ... 18

Figure 23. Illustrations of the shroud emission electron orbits (red) and the velvet electrons (blue)........... 18

Figure 24. Lapostelle normalized emittance at $\mathrm{t}=41 \mathrm{~ns}$ with/without shroud emission........................... 18

Figure 25. Electron orbits are shown for simulated no emission from two different areas of the cathode... 20

Figure 26. Emittance is shown as a function of axial position for different bands of non-emission. ........... 20

Figure 27. Images of velvet cathode during emission are shown during rise time (left) and "flat top". ...... 20

Figure 28. X-Y plots of orbits immediately in front of the cathode for two different simulations.............. 21

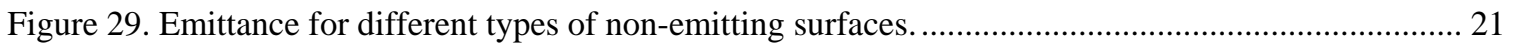

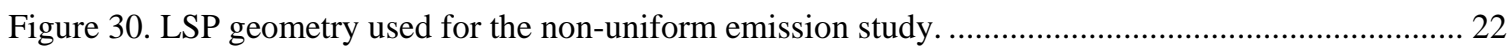

Figure 31. Lapostelle normalized emittance at $\mathrm{z}=50 \mathrm{~cm}$ for uniform (left), non-uniform (right) emission.22

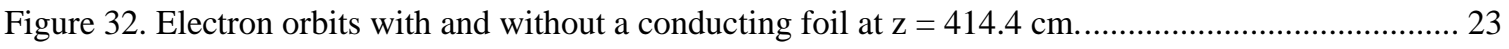

Figure 33. Beam emittance versus time at $\mathrm{z}=414 \mathrm{~cm}$ with/without a grounded, conducting foil.............. 23

Figure 34. Emittance as function of axial position with/without a grounded, conducting foil at $180 \mathrm{~cm}$.... 24 


\section{Introduction}

A modeling effort was undertaken to characterize the injector of the Flash X-Ray accelerator (FXR) at Site 300 and determine what steps could be taken to optimize its performance. This report summarizes several FXR Notes ${ }^{1,2,3}$ issued as part of this effort. The motivation for the modeling effort was the larger than expected beam emittance. Emittance is an important beam parameter for accelerators used for radiography since the minimum achievable $\mathrm{x}$-ray spot size (resolution) is proportional to the square root of the emittance. Table 1 lists the measured emittance for several high-current induction accelerators including the cathode type and measurement technique. A report by Art Paul ${ }^{4}$ contains a similar table. The "Scaled" emittance in Table 1 is defined as:

$$
\varepsilon_{\text {Scaled }}=\varepsilon_{N} \sqrt{\frac{I_{F X R}}{I_{\text {accelerator }}}} \text {, where } I_{\text {accelerator }} \text { is the accelerator current for the respective measurements. }
$$

This scaling adjusts for the effect of current on emittance assuming the same beam radius. Two conclusions can be drawn from the table. First is that the emittance is a factor of four or more higher at the end of the FXR accelerator than for other similar accelerators. Second is that the emittance starts high from the exit of the injector. The 2.1-MeV measurement for FXR was performed at the injector exit.

Note that in Table 1, the emittance given is a normalized, rms emittance. For the accelerators listed, the beams are nominally circular in cross section and the figure of merit for their radiographic performance is the radius of the beam envelope at the Bremsstrahlung converter, i.e. the spot size, to which the beam can be focused. Thus the rms emittance defined by Lee and Cooper ${ }^{5}$ for use in the envelope equation is more pertinent than the more general edge emittance of Lapostolle ${ }^{6}$. If the current distribution is known, the two emittances can be related. Also, most measurements of beam emittance are calculated from the data as rms values. Another caveat is that subtleties in the implementation of the emittance definition during analysis of orbit distributions, whether simulated or measured, can affect the answer. We have tried to use equivalent definitions for emittances given in Table 1, but it would be prudent to assume large error bars. After publishing the FXR Notes given in References 1, 2, and 3, it was discovered that the algorithm for calculating the emittances for our simulations omitted an azimuthal component. Rechecking the data indicated that the published emittances were approximately 15\% low depending on the code used for the simulation. All graphs and figures in this summary report are taken directly from these references and include the error unless otherwise stated. However, the parameter studies were relative comparisons unaffected by this error and the error is small compared to the uncertainties with actual measurements.

Table 1. Measured emittance for high current linear induction accelerators.

\begin{tabular}{|l|l|l|c|l|c|c|}
\hline Accelerator & $\begin{array}{c}\text { Cathode } \\
\text { Type }\end{array}$ & $\begin{array}{c}\text { Energy } \\
(\mathrm{MeV})\end{array}$ & $\begin{array}{c}\text { Current } \\
(\mathrm{kA})\end{array}$ & $\begin{array}{c}\text { Type } \\
\text { Measurement }\end{array}$ & $\begin{array}{c}\text { Normalized } \\
\varepsilon_{N, r m s}(\mathrm{~cm}-\mathrm{mr})\end{array}$ & $\begin{array}{c}\text { Scaled } \\
\boldsymbol{\varepsilon}_{N, r m s}(\mathrm{~cm}-\mathrm{mr})\end{array}$ \\
\hline ARIX $^{7}$ & Velvet & 3.85 & 2.0 & Envelope & 110 & 115 \\
\hline ETA-II $^{8}$ & Dispenser & 2.55 & 1.4 & Pepper Pot & 61 & 93 \\
\hline ETA-II & Dispenser & 6.0 & 2.0 & Pepper Pot & 47 & 60 \\
\hline $\begin{array}{l}\text { DARHT- } \\
\mathrm{I}^{9}\end{array}$ & Velvet & 3.75 & 4.0 & Envelope & 75 & 370 \\
\hline FXR $^{11}$ & Velvet & 17.5 & 3.3 & OTR & 370 & 400 \\
\hline FXR $^{12}$ & Velvet & 17.9 & 3.3 & Envelope & 400 & 193 \\
\hline FXR & & 3.3 & Pepper Pot & 193 & 36 \\
\hline FXR & Velvet & 2.1 & 3.3 & Simulation & 36 & 67 \\
\hline
\end{tabular}

A related issue to the larger than expected beam emittance is the difficulty in simulating the measured beam radius for different transport conditions. For example, the radius of the FXR beam can be measured at a fix location as a function of the field of an upstream focusing solenoid. The measured data are subject to many uncertainties such as absolute beam energy, relative energy flatness, absolute magnetic field, and diagnostic characteristics. Even allowing large error bars for the measurements, past simulations have done a poor job of predicting the radius. This is especially true for solenoid and/or measurement locations early in the accelerator where space charge dominates. 
Advances in computers and codes in recent years provided encouragement to revisit the design of the FXR injector to identify potential techniques for lowering the emittance. The present diode package was designed with the venerable EGUN ${ }^{14}$. EGUN has been the primary gun design code and is regularly used to benchmark newer gun codes. However, it has several limitations when used for the FXR injector optimization studies. The first can be thought of as an aspect ratio restriction on the solution area. Electron orbits can only be calculated along the axis to a distance equal to approximately five times the beam transport diameter before numerical instabilities impact the results. In the case of the FXR injector, it is necessary to continue the calculation to approximately 15 diameters for a suitable point to transfer the orbit information to a beam transport code or 40 diameters to reach the exit of the injector. The longer distances are crucial if simulations are to be compared to measurements. EGUN is also an axially symmetric gun code. Issues involving misalignments that break the axial symmetry cannot be addressed. Finally, EGUN is a time-independent code so that studies of the beam head where the energy is increasing to the steady state or "flat top" value cannot be performed.

The TriComp ${ }^{15}$ series of finite-element software for electromagnetic solutions including charged particle transport was used for time-independent, axially symmetric parameter studies. BSTAT, the TriComp magnetostatic solver, was crossed checked with Poisson ${ }^{16}$ then used to create the magnetic input files used by the other codes in the parameter study. TRAK, another code of the TriComp suite, is a ray tracing charged particle optics code similar to EGUN. The fastest running of the particle codes used, TRAK generated the nominal case for comparison with the other codes. Figures and graphs given in this report that were generated by the TriComp codes are taken from Reference 3 unless otherwise noted. For timeindependent, three-dimensional, parameter studies, the AMAZE ${ }^{17}$ series of three-dimensional, finiteelement software for electromagnetic solutions including charged particle transport was used. Figures and graphs given in this report that were generated by the AMAZE codes, e.g. OmniTrak, are taken from Reference 1. LSP ${ }^{18}$, a three-dimensional, electrodynamic, Particles-In-a-Cell (PIC) code was used to study time dependent phenomena. Due to the large computational resources needed for simulating time dependent phenomena, LSP was used primarily in its axially symmetric mode and limited to cylindrical coordinates for three-dimensional studies. Figures and graphs given in this report that were generated by LSP are taken from Reference 2 with the exception of movie frames taken from an unpublished presentation by D. Blackfield.

Transport simulations beyond the exit of the injector and comparison to measurements of the beam envelope were not a part of the parameter study. However, output from TRAK was used as input for both TRANSPORT $^{19}$ and AMBER ${ }^{20}$. TRANSPORT works well when emittance is conserved and the current distribution remains relatively constant. AMBER simulations allow for changes in emittance and current distribution. Using TRAK output, AMBER simulated the beam from $150 \mathrm{~cm}$ to $400 \mathrm{~cm}$. The result of the AMBER simulation was then compared to a TRAK simulation of the beam to $400 \mathrm{~cm}$ to check consistency between the two codes ${ }^{21}$.

\section{Electric Field on Diode}

The parameter study began with calculating the electric field in the diode region. This effort required the mechanical geometry of the diode and the applied pulsed power voltage. The two time independent gun codes, TRAK and OmniTrak, used only the "flat top" voltage value. ESTAT was used to establish the nominal electric fields that were then compared with results from HiPhi and LSP. Figure 1 shows a photograph of the diode and the simplified diode geometry used for the study. The center of the bucking coil was used as the longitudinal zero reference. The longitudinal position of the emitting surface was located at $3 \mathrm{~cm}$ with the anode tip at $15 \mathrm{~cm}$ in this reference frame. Refer to the individual reports (References 1, 2, and 3) for details on the simulated geometry. The actual geometry was checked after the start of the study and was found to vary slightly from that shown ${ }^{22}$. Since the changes to the geometry did not appear to have a significant effect on the calculated emittance and we were studying relative changes, the referenced geometry for the parameter study was maintained as the geometry shown in Figure 1. Figure 1 also shows the equipotential lines at the "flat top" or steady state voltage. 
Figures 2 and 3 show details of the longitudinal and radial electric field components at several axial positions immediately in front of the cathode. Note that $\mathrm{z}=3.0 \mathrm{~cm}$ is the location of the emitting surface. The field stress on the emitting surface varies from $140 \mathrm{kV} / \mathrm{cm}$ at the center to $80 \mathrm{kV} / \mathrm{cm}$ at the outer radius $(r=5.4 \mathrm{~cm})$. Miller ${ }^{23}$ conducted studies on velvet cathodes that indicated the threshold electric field strength for surface flashover of the velvet is approximately $16 \mathrm{kV} / \mathrm{cm}$. He listed other velvet cathode experiments that used field stresses from $35 \mathrm{kV} / \mathrm{cm}$ up to $180 \mathrm{kV} / \mathrm{cm}$. Thus, the FXR injector cathode field stress appears sufficient to generate plasma on the velvet surface of the cathode and extract space charge limited electron emission. A higher field stress than the $80-140 \mathrm{kV} / \mathrm{cm}$ may be required to produce a uniform, low emittance beam, as will be discussed in the section on Non-Uniform Emissions.
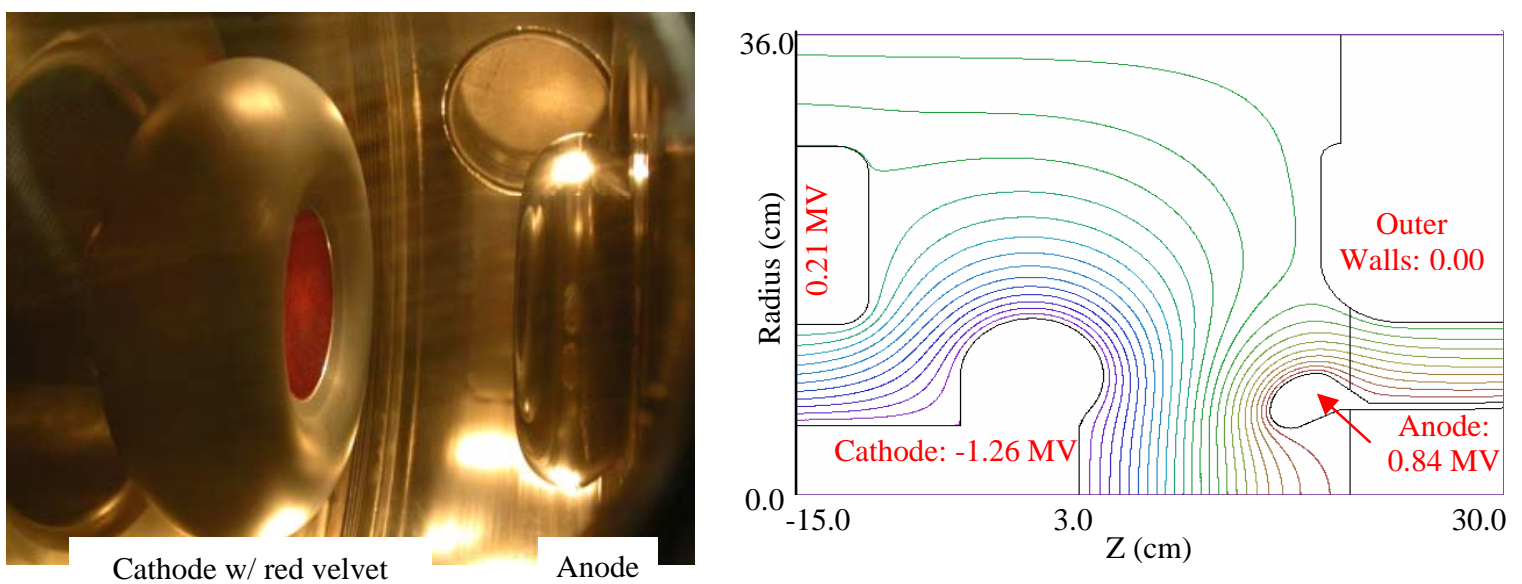

Figure 1. Photograph of the diode region and simulated geometry with equipotential line.

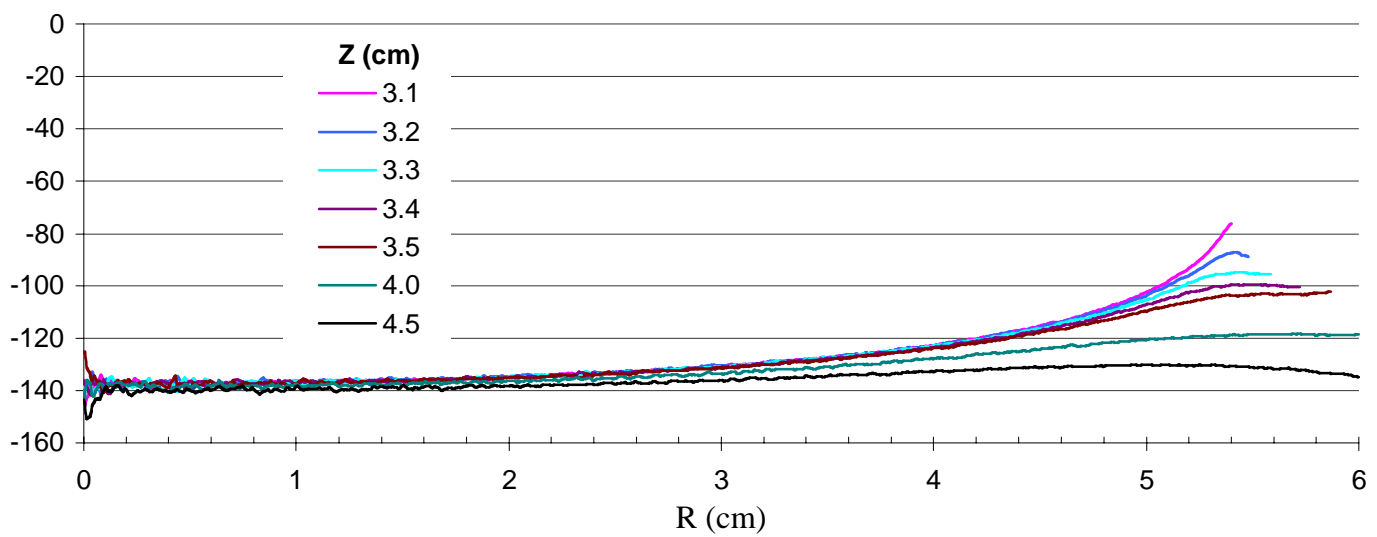

Figure 2. The longitudinal electric field plotted as a function of radial position at different axial positions. 


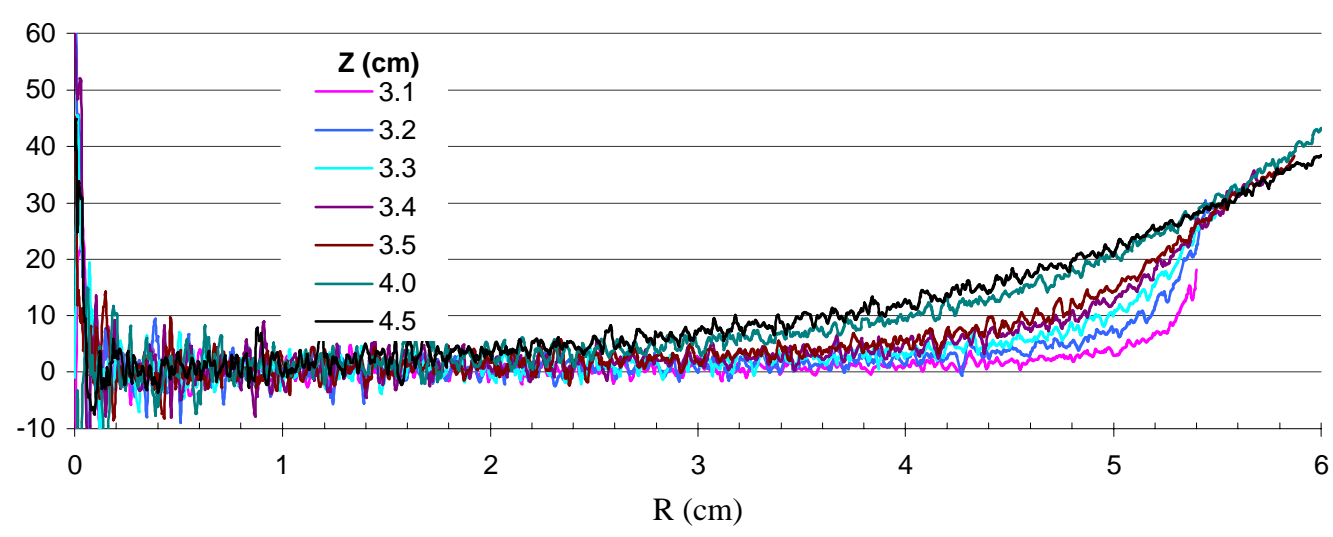

Figure 3. The radial electric field plotted as a function of radial position at different axial positions.

The radial field at the emission surface is important for low emittance and initial focusing of the beam. Ideally it would be zero once beam space charge effects are included if the beam is to have a uniform distribution parallel to the axis. Thus, the small radial component of the applied field towards the outer radius, shown in Figure 3, serves to balance space charge forces and provides some inward focusing in the distance between the cathode and the anode, the A-K gap, until the magnetic field focusing becomes effective. The magnetic field is "bucked out" or zeroed as much as possible at the cathode to prevent the beam from having a canonical angular momentum. Refer to the subsection on the Bucking Coil in the Section Effect of Misalignment and Improper Bucking.

\section{Magnetic Fields in the Injector}

Without focusing fields the space charge forces between the extracted electrons would cause the beam to expand with electrons intercepting the walls of the injector. The FXR injector uses a series of solenoids to control the trajectory of the electrons. Figure 4 shows the location of the solenoids and constant $A_{z}$ contours used in the study. There are 10 solenoids in the injector. The fields of the transport solenoid (I-35) at the exit of the injector and the solenoid located in the first accelerator cell (J-21) were also included as their fields extended into the injector. The total magnetic field for different radial positions is shown in Figure 5.

Early in this study we noticed that the combination of the solenoid geometries and currents used during operations did not produce the expected low magnetic flux through the emitting surface in the simulations. Diamagnetic loop (DML) measurements ${ }^{24}$ indicated that the flux at the cathode surface was actually much smaller than the simulations values. For the simulations to achieve a flux free cathode, the I-20 solenoid (bucking coil) current was increased by $15 \%$. Careful measurements of the injector geometry and magnetic fields in the diode region were taken to resolve the issue. Discrepancies in the location of the bucking coil and permeable material were found that accounted for a portion of the error in calculating the magnetic field at the cathode. Upon revisiting the BSTAT simulations with corrected geometry and magnetic field data, we discovered that the radial extent of the solution boundary had to be extended by a factor of three, out to 3 meters to avoid axial distorting the magnetic field near the axis. Fortunately using this earlier inaccurate magnetic field data produced only small changes to the simulated beam envelope and had no significant effect on the emittance. Rather than repeat the rather lengthy and time-consuming parameter simulations, we chose to continue using the original magnetic field calculations to finish the study. TRAK was then used with the corrected geometry and magnetic field for comparison with actual beam measurements.

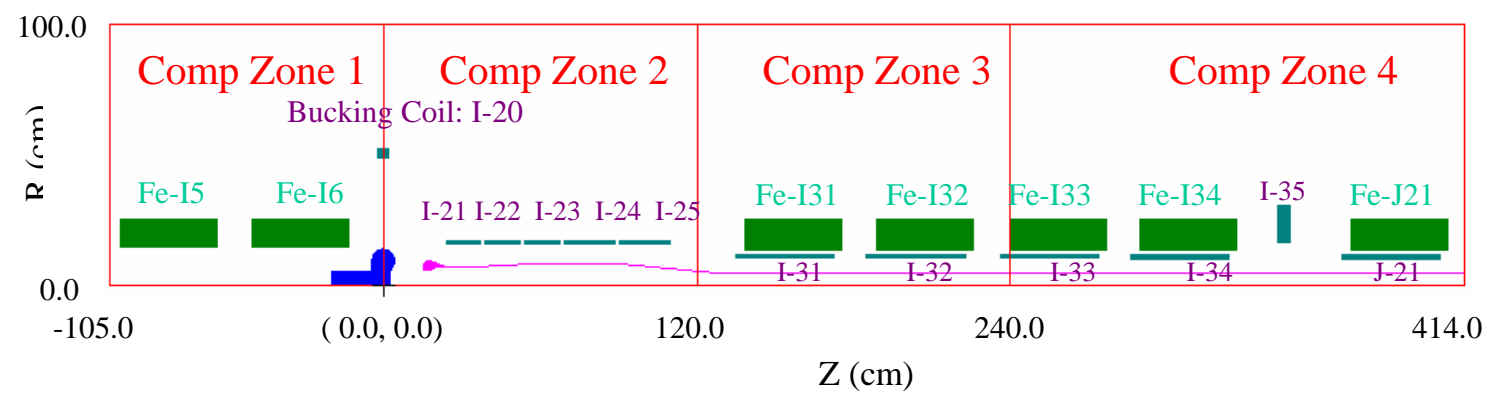




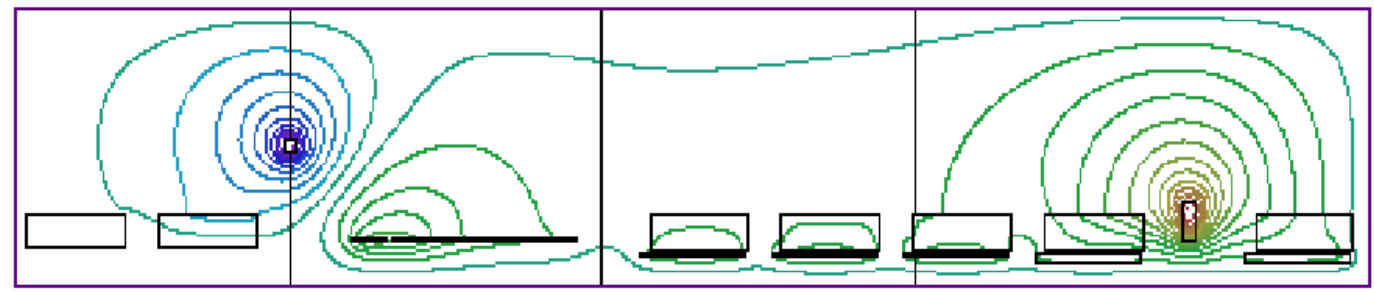

Figure 4. Layout of the injector solenoids with constant $A_{z}$ contours.

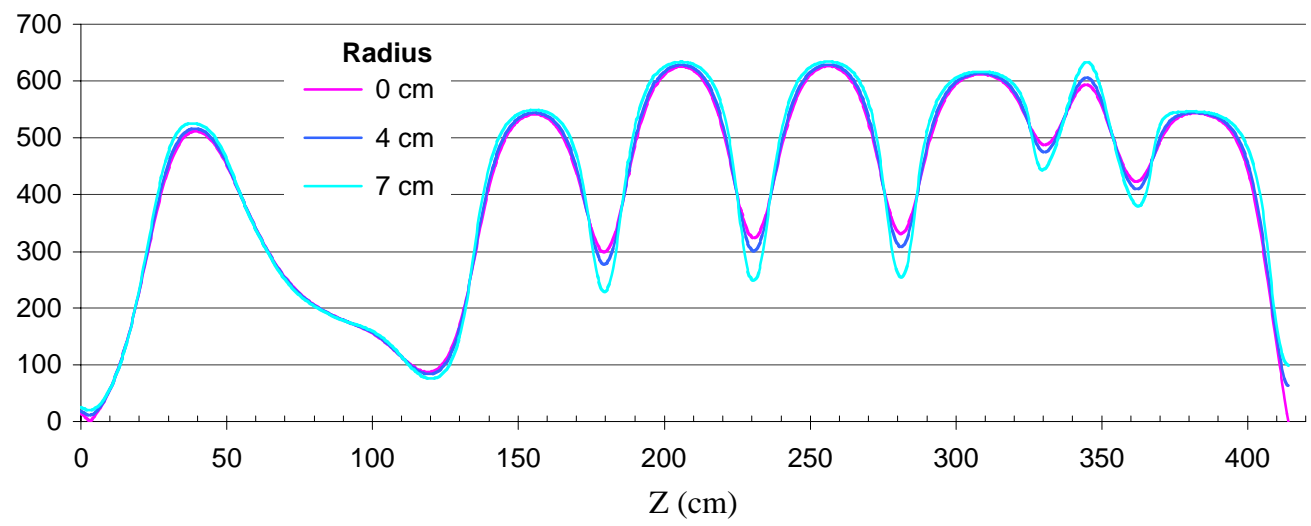

Figure 5. The magnetic field is plotted as a function of longitudinal position for different radial distances.

\section{Emission Model and Nominal Case}

\section{Space Charge Limited Emission}

Space charge limited emission was assumed with all the gun codes. By that we mean that we assumed the velvet cathode surface flashed over generating a plasma. The extraction of electrons from this plasma was Child-Langmuir law limited. Table 2 lists the calculated current. Figure 6 shows the orbits produced by TRAK. The simulations produced approximately $3.4 \mathrm{kA}$ of current in agreement with the nominal $3.3 \mathrm{kA}$ measured at the exit of the injector.

Table 2. Emission current calculated by the various beam codes with the mesh symmetries used.

\begin{tabular}{|l|c|c|}
\hline \multicolumn{1}{|c|}{ Code } & Mesh Symmetry & Steady State Current \\
\hline TRAK & 2-D Cylindrical & $3.47 \mathrm{kA}$ \\
\hline LSP & 2-D Cylindrical & $3.3 \mathrm{kA}$ \\
\hline LSP & 3-D Cylindrical & $3.4 \mathrm{kA}$ \\
\hline OmniTrak & 3-D Cartesian & $3.22 \mathrm{kA}$ \\
\hline Average & & $3.35 \pm 0.11 \mathrm{kA}$ \\
\hline
\end{tabular}

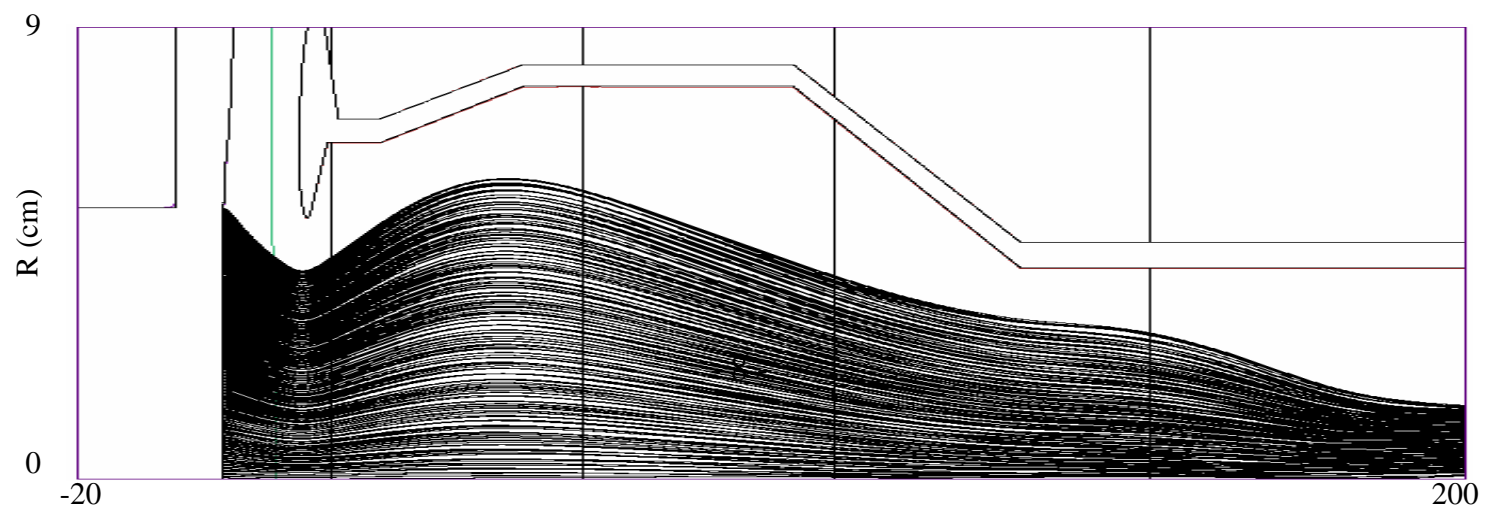


Figure 6. TRAK simulated electron orbits for the FXR injector.

\section{Thermal Emittance}

The intrinsic or thermal emission of the velvet cathode must be included in the simulation. TRAK and OmniTrak assign a random-uniformly weighted angular displacement of the orbits in three-dimensional space about the perpendicular to the emission surface. The maximum angular displacement is input to the code and determines the thermal spread. LSP allows an input of transverse energy given in $\mathrm{eV}$. A onedegree random polar angular energy spread on the initial orbit trajectories was used for the TRAK and OmniTrak nominal cases to simulate the thermal temperature of the cathode. A study ${ }^{25}$ on beam emittance of a field emission diode similar to the FXR injector diode indicated average spread angles of $14 \mathrm{mrad}$ (normalized), about half of our nominal value. Art Paul ${ }^{26}$ has performed some theoretical calculations indicating a slightly higher value. LSP used a $2 \mathrm{eV}$ thermal energy.

OmniTrak was used to simulate three different values for the thermal temperature represented by angular spreads of 0 degrees (cold beam), 1 degree (nominal case), and 2 degrees. The radius was very similar for the three cases. The difference in the rms radii was less than $1 \%$ and the maximum radius differed by less than $2 \%$ (Cold beam or 2 degrees from the nominal case) measured at $100 \mathrm{~cm}$. Each degree of angular spread added approximately $10 \%$ to the value of the emittance after an axial position of approximately 70 $\mathrm{cm}$. The one-degree value for the nominal case is most likely within $\pm 1 / 2$ degree of the correct value so thermal emittance was not considered a significant factor to overall beam emittance. Figure 7 shows the change in trace space immediately in front of the cathode for the different values of temperature. Emittance as a function of position along the axis is given in Figure 8 for the three values of initial angular spreads.
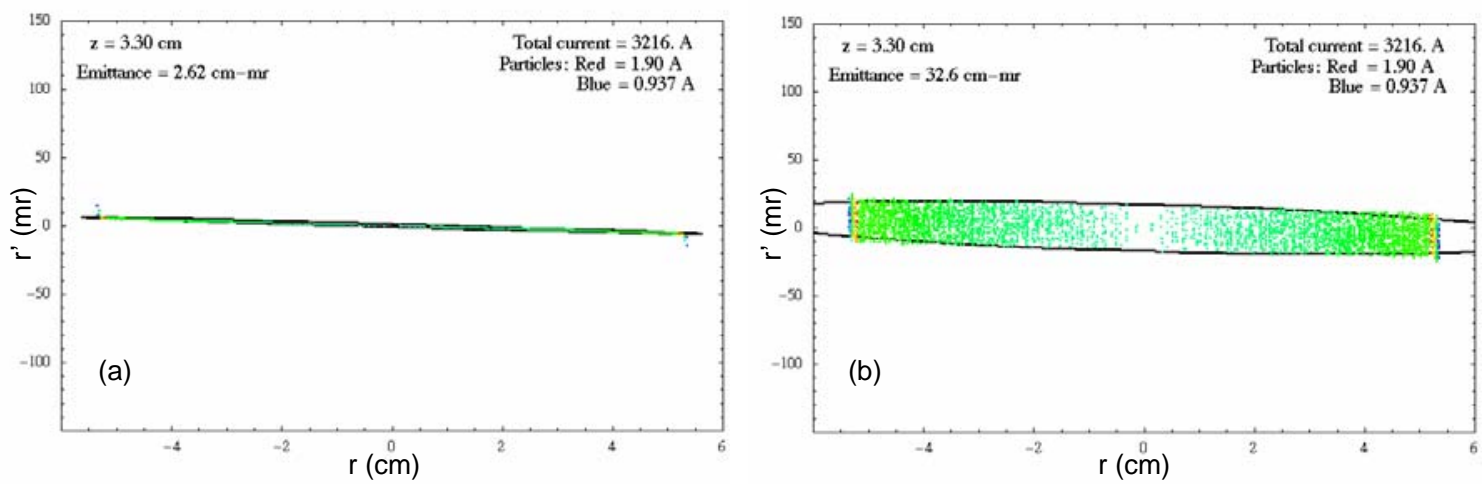

The three views of trace space are for the cases of (a) no thermal spread, (b) $1^{\circ}$ random angular spread, and (c) $2^{\circ}$ angular spread. Note that the radius of the distribution does not change, only the angle. Each degree of angular spread increases the emittance approximately $30 \mathrm{~cm}-\mathrm{mr}$ ( $5 \mathrm{~cm}-\mathrm{mr}$ normalized).

Figure 7. Trace space in front of the cathode.

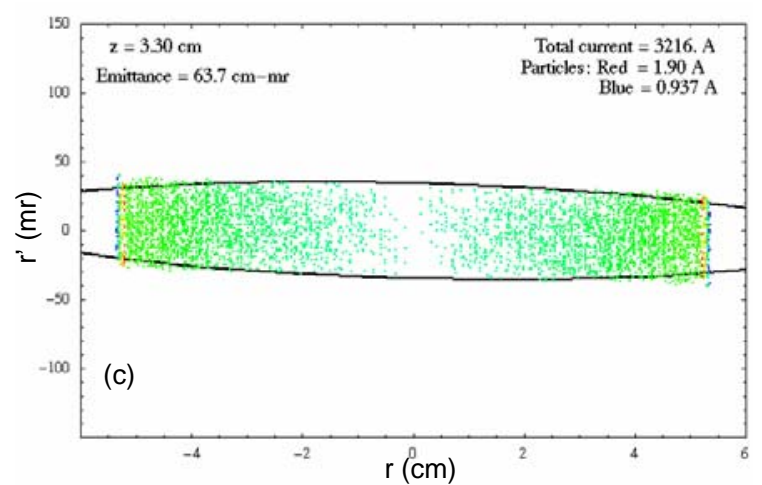




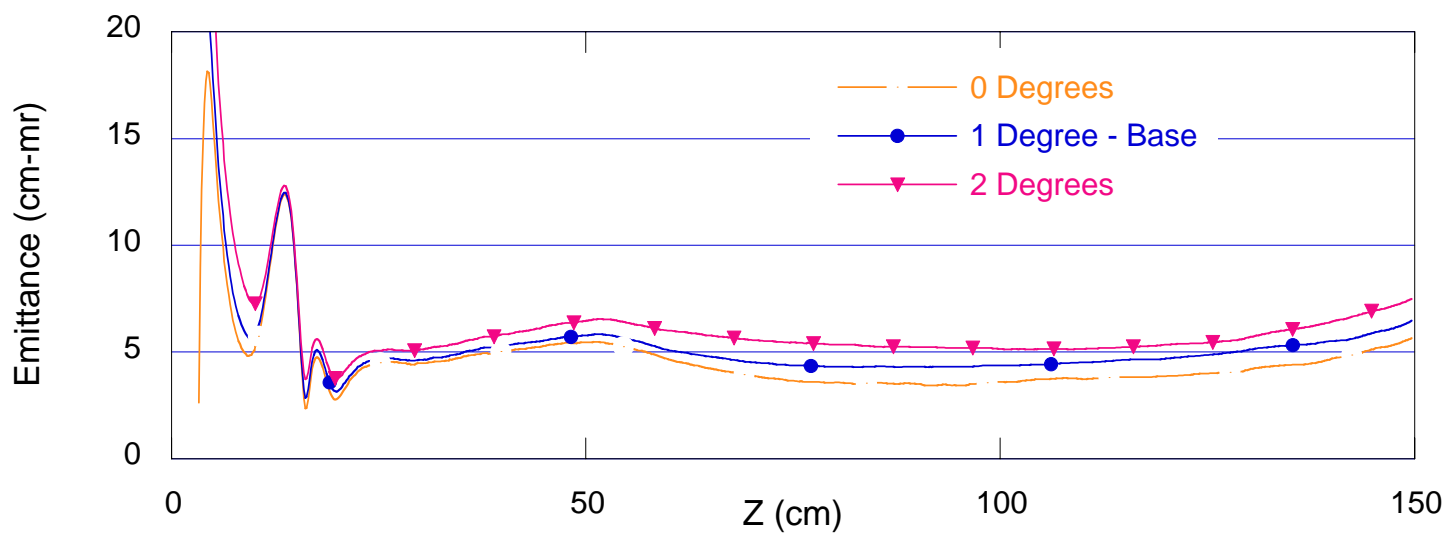

Figure 8. Emittance is shown as a function of axial position for three different angular spreads.

\section{Time Dependent Emission and Transport}

Time dependent emission was not a part of the parameter study, but the information was generated as part of the LSP simulations. Figure 9 is a set of time snapshots (movie frames) of the beam as it emerges from the cathode and moves through the injector. A linear time ramp to full voltage at 6 ns was used. The electrons at full voltage take about $14 \mathrm{~ns}$ to transverse the length of the injector. Approximately $22 \mathrm{~ns}$ after first emission the beam envelope is at the steady state value. Figures 9 (d) and (e) can be compared with Figure 6 as the steady state value up to $2 \mathrm{~m}$ is reached in $13 \mathrm{~ns}$. The lower energy electrons during the rise time of the energy are not properly matched to the solenoid focusing and are "sprayed" along the inside of the injector. By the exit of the injector most of these early electrons are lost resulting in a steeper rise time for the pulse in the accelerator.

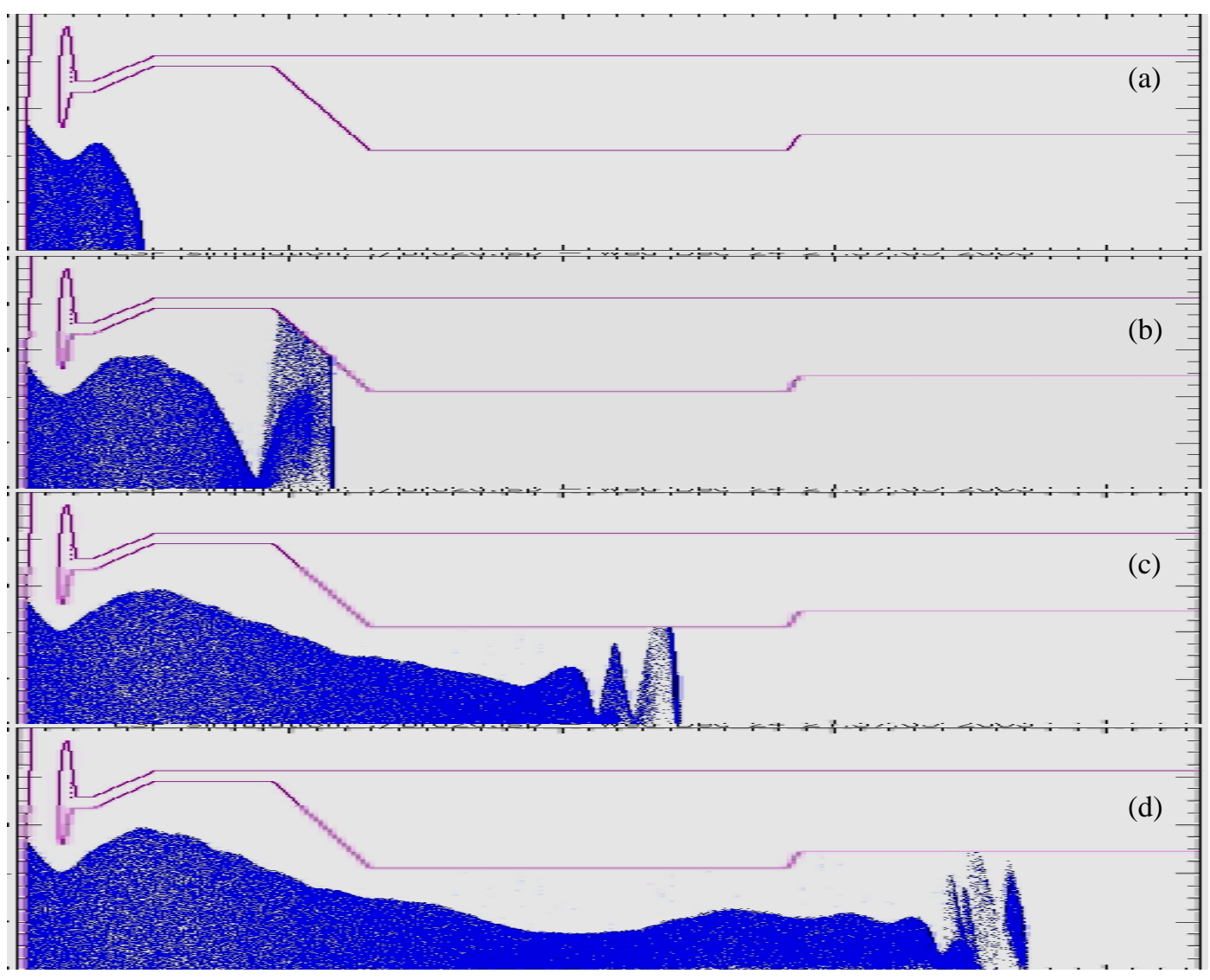




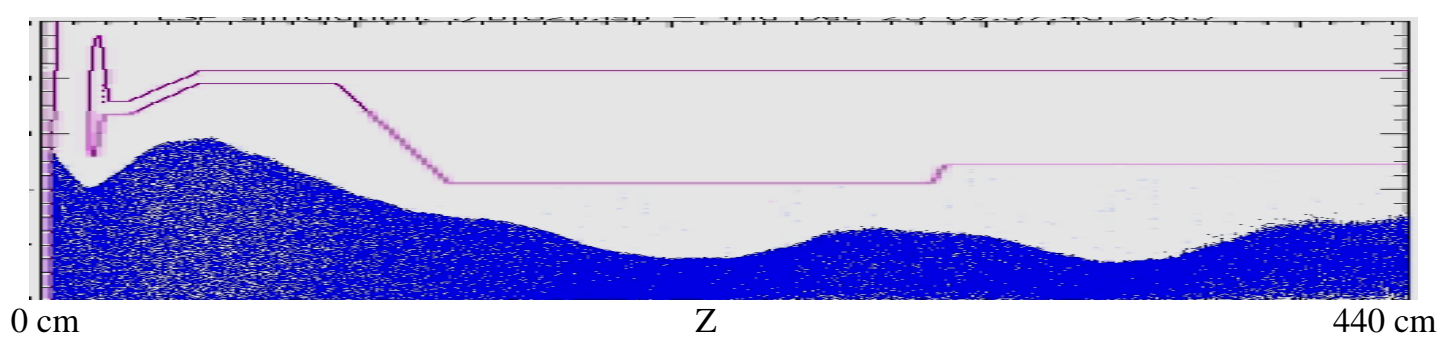

Figure 9. Snapshot of the beam at five different times into the pulse:

(a) $1.5 \mathrm{~ns}$, (b) $4 \mathrm{~ns}$, (c) $8.5 \mathrm{~ns}$, (d) $13 \mathrm{~ns}$, and (e) $22 \mathrm{~ns}$ after first emission.

\section{Comparison of TRAK, OmniTrak, and LSP}

The three codes agreed closely on the beam radius and current density. Figure 10 is a comparison of the calculated rms radius. The LSP simulation was two-dimensional. The OmniTrak simulation appears to loose accuracy after approximately $150 \mathrm{~cm}$. The beam envelope for the nominal FXR injector parameters is dominated by space charge and relatively insensitive to emittance.

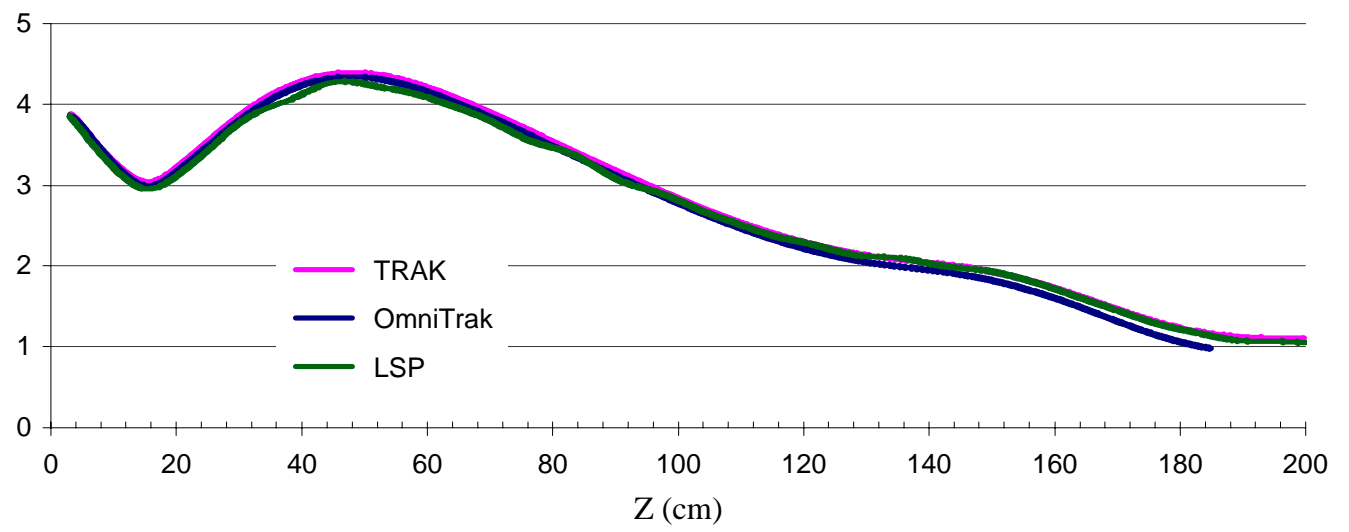

Figure 10. Comparison of the calculated rms radius between TRAK, OmniTrak, and LSP.

Emittance is very sensitive to small changes in orbit angle. Figure 11 is a comparison of the calculated emittance. The OmniTrak and TRAK traces have similar shapes for the first 30 to $40 \mathrm{~cm}$. The vertical offset is due to the coarser grid used for OmniTrak. The coarser grid also is the likely explanation for the difference between LSP and OmniTrak after $20 \mathrm{~cm}$. The most interesting feature of Figure 11 is the "hump" in the TRAK trace between the axial positions of 50 and $150 \mathrm{~cm}$ where the other codes predict nearly constant emittance.

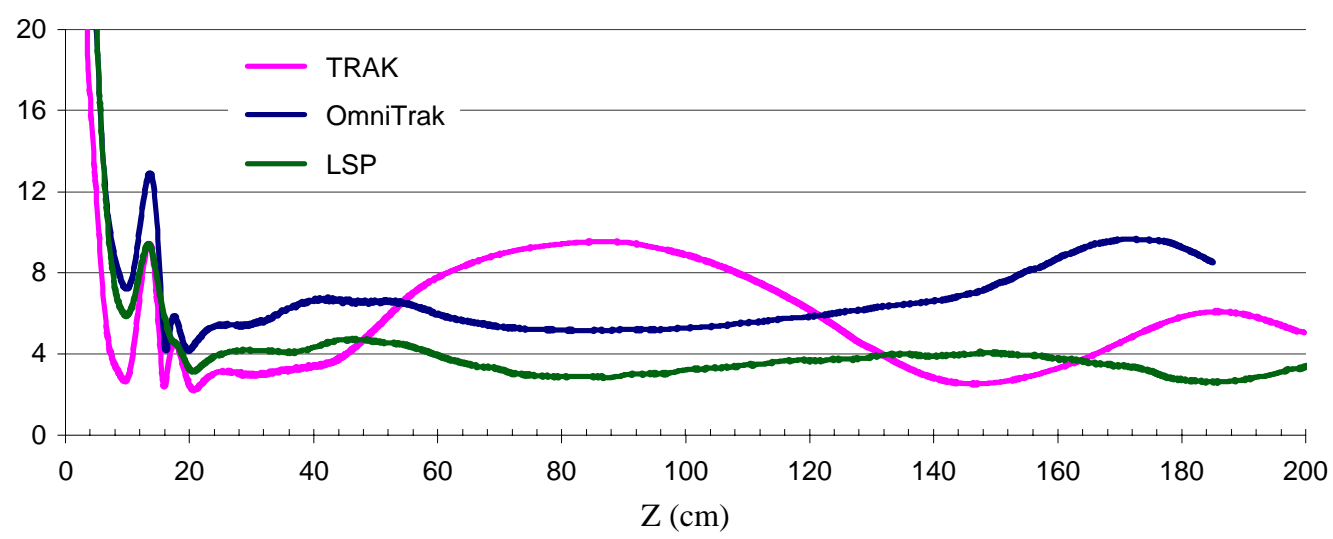

Figure 11. Comparison of the calculated emittance between TRAK, OmniTrak, and LSP. 


\section{Effects of misalignments and improper bucking}

Simulations were performed to see the effect of errors in aligning the diode or adjusting the bucking coil on the emittance. These simulations determine the tolerance requirements for the FXR injector. Table 3 lists the results for the various simulations. More details are given below.

Table 3. Effect of Alignment and Bucking Coil errors on Emittance

\begin{tabular}{|l|l|l|l|}
\hline Error Type & Comment & Error Magnitude & \multicolumn{1}{|c|}{ Emittance Increase } \\
\hline \multirow{2}{*}{ Cathode surface to shroud } & $\begin{array}{l}\text { Absolute change in } \varepsilon_{N} \\
\text { is more relevant. }\end{array}$ & $\pm 2 \mathrm{~mm}$ & $5 \mathrm{~cm}-\mathrm{mr}(5-30 \%)$ \\
\cline { 3 - 4 } & $\begin{array}{l}\text { Emittance decreased } \\
\text { A-K Gap Spacing }\end{array}$ & $\pm 4 \mathrm{~mm}( \pm 3 \%)$ & $15 \mathrm{~cm}-\mathrm{mr}(20-120 \%)$ \\
\hline Cathode Tilt & & $10 \mathrm{milliradians}$ & negligible \\
\hline Cathode Offset & $\begin{array}{l}\text { Emittance increase varies } \\
\text { with axial location }\end{array}$ & $4 \mathrm{~mm}$ & $<30 \%$ \\
\cline { 3 - 4 } & & $8 \mathrm{~mm}$ & $70 \%$ \\
\hline Bucking Coil & & $\pm 30 \%$ & negligible \\
\hline
\end{tabular}

\section{Emission surface relative to shroud}

The longitudinal position of the emission surface (velvet) relative to the cathode shroud was varied over a range of $\pm 4 \mathrm{~mm}$. If the emission surface protrudes beyond the edge of the cathode shroud, the field stress on the outer radius of the velvet will increase leading to higher current density with a larger outward radial component. Similarly, if the velvet is retracted into the cathode housing, the field stress will be reduced at the outer radius and have more of an inward radial component. It was found that the current changed by approximately $\pm 3.5 \%$ per mm shift (plus for protruding). Either scenario is undesirable as it leads to orbits with non-similar motion, i.e. higher emittance. Emittance as a function of longitudinal position for the various simulations is shown in Figure 12. The simulations developed different phase advances for the emittance making it difficult to give a relative change in emittance. Around $150 \mathrm{~cm}$, the emittance is at a minimum and the relative increase is $30 \%(120 \%)$ for $\pm 2 \mathrm{~mm}( \pm 4 \mathrm{~mm})$. This can be compared to the $90 \mathrm{~cm}$ position where emittance is at a local maximum and the relative increase is $5 \%(20 \%)$ for $\pm 2 \mathrm{~mm}( \pm 4 \mathrm{~mm})$. A more consistent method of describing the emittance change is that it is approximately a $5 \mathrm{~cm}-\mathrm{mr}(15 \mathrm{~cm}-$ $\mathrm{mr}$ ) increase in the normalized emittance for a $\pm 2 \mathrm{~mm}( \pm 4 \mathrm{~mm})$ shift.

A change of $1 \mathrm{~mm}$ in the position of the velvet with the cathode shroud is easily observed. A more difficult question is the location of the plasma with respect to the surface of the velvet and whether that is the more important location. The simulations indicate that a relatively large shift in location has a small impact on the absolute value of the emittance. The pepperpot measurements taken at the end of the injector were done with different shifts in the cathode surface and no measurable change was noted in the emittance. The rms radius varied $\pm 2.5 \%$ per mm shift (plus for protruding).

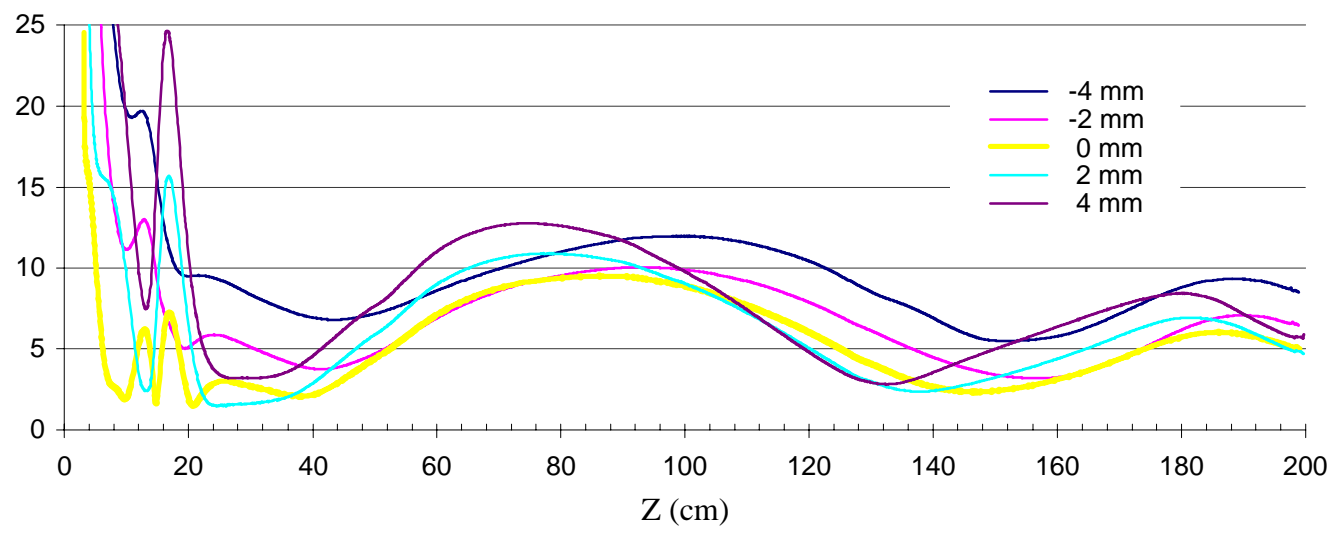

Figure 12. Emittance is shown as a function of axial position for different cathode/shroud spacing. 


\section{Variation in A-K gap}

The distance between the cathode and the anode, the A-K gap, can also be varied in the FXR injector. A number of simulations were performed to determined the effect of errors in setting this distance. Results of the simulations are displayed in Figure 13. The nominal gap for injector operations is $12.0 \mathrm{~cm}$. The emittance had a small increase, less than $10 \%$, for the smaller gap and improved slightly (decreased) for the longer gaps. The emitted current varied $\pm 5 \%$ as expected for the smaller/larger gap spacing. A mechanical fixture is used for adjusting the A-K gap distance. An external reference guage on the cathode stalk allows monitoring of adjustments to the A-K gap during operations.

\section{Cathode tilt}

A second type of diode misalignment is the rotation of the emitting surface with respect to the plane of the anode, i.e. tilt. Two different tilts of the cathode were compared to the perfectly aligned nominal case. The change in radius, both rms and maximum, was negligible for the two tilts. Emittance for the two tilts compared to the nominal case is shown in Figure 14. Tilts of 10 mrad have little or no effect on beam quality. Intuitively, this is not unexpected. The electric field at the emission surface is tilted with respect to the $\mathrm{z}$-axis as it is perpendicular to the conducting emission surface. However, the electric field reorients to the "global" geometry very near the surface and looks similar to the situation of a transverse shift of approximately $0.2 \mathrm{~mm}$. This is too small of a shift to be resolved by the grid leading to an electric field that closely resembles that of the nominal case. The orbits (electrons) follow the electric field lines since there is little external magnetic focusing field near the cathode.

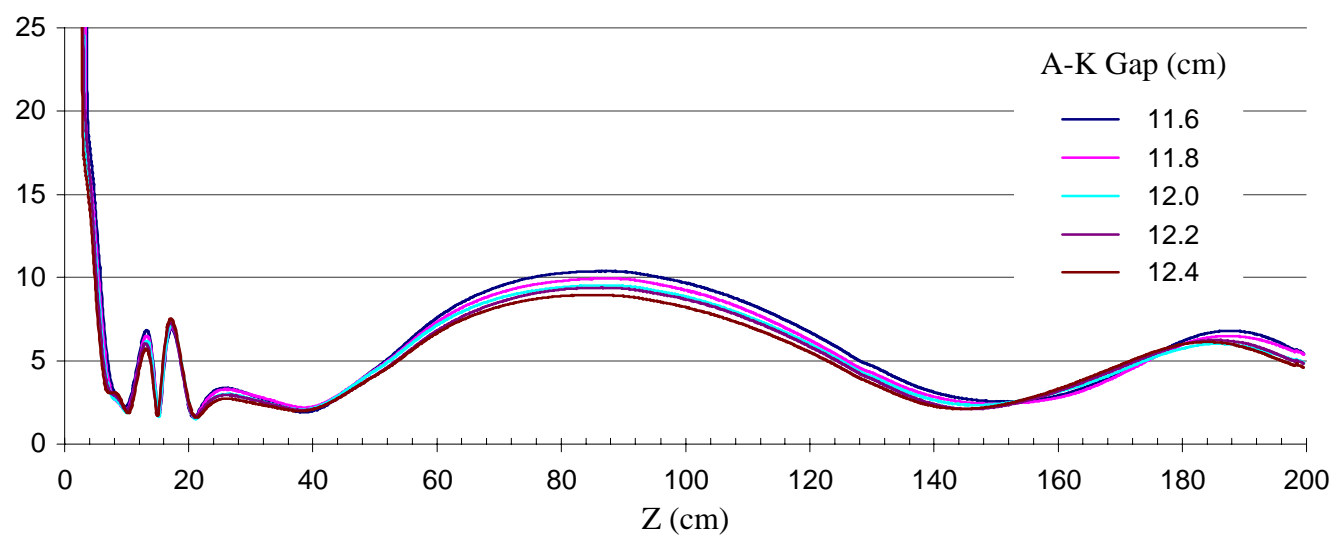

Figure 13. Emittance is shown as a function of axial position for different A-K spacing.

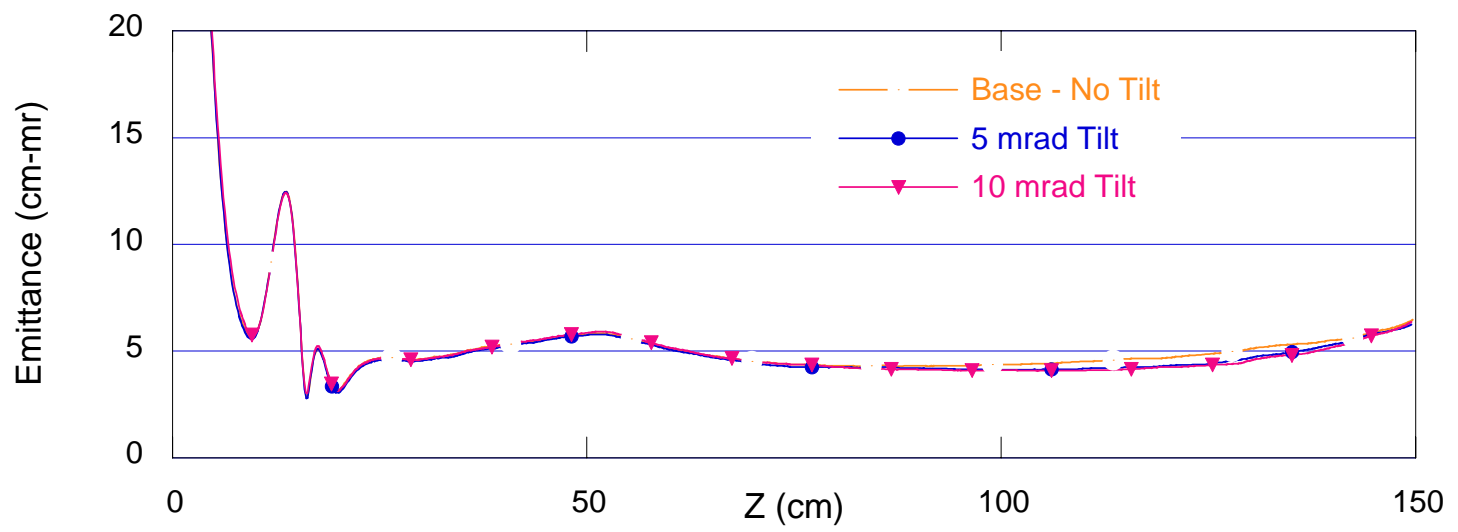

Figure 14. Emittance is shown for different amounts of cathode tilt with respect to the axis. 


\section{Cathode offset}

Transverse misalignment of the diode is another possible source of beam degradation. Two different transverse offsets of the cathode were compared to the perfectly aligned nominal case. The cathode stalk and emitting surface were offset $4 \mathrm{~mm}$ and $8 \mathrm{~mm}$ for the two simulations.

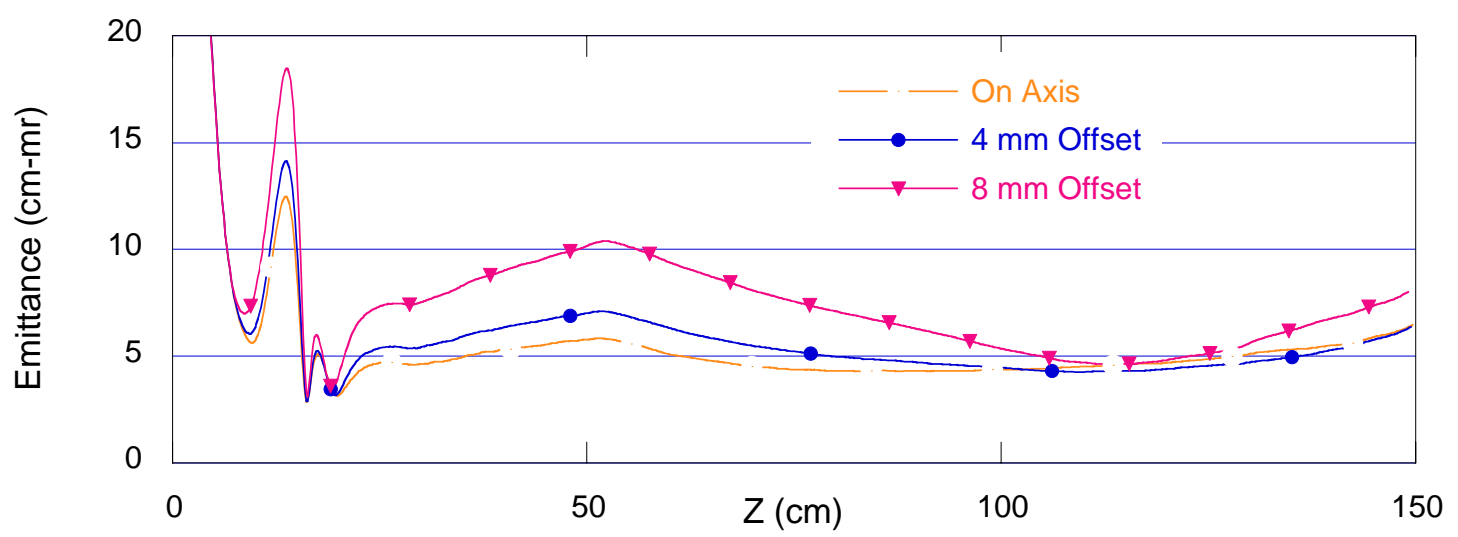

Figure 15. Emittance for different transverse offsets of the cathode from the z-axis.

Beam radius with respect to the centroid changed by less than $2 \%$ for the rms radius and less than $3 \%$ for the maximum radius measured at $100 \mathrm{~cm}$ for the two cases with respect to the nominal case. The emittance for the 4-mm offset varied from a few percent to as much as $30 \%$ with respect to the nominal case depending on where it was viewed axially. Refer to the plot of emittance versus axial position in Figure 15. However, the emittance for the 8-mm offset deviated significantly from the nominal case for most positions. Diode alignment should be held to less than $4 \mathrm{~mm}$ offset to avoid noticeable beam degradation.

\section{Bucking coil field strength errors}

The actual magnetic flux on the cathode was of interest for two reasons. As noted above, we had to increase the calculated bucking field by $15 \%$ to achieve the measured flux on the cathode. The cathode flux contribution to the beam envelope is similar to that of the emittance. One could define an effective emittance that could be substituted in the envelope equation in place of the emittance and flux terms:

$$
\varepsilon_{N, \text { eff }}^{2}=\varepsilon_{N, r m s}^{2}+\frac{P_{\theta}^{2}}{c^{2}}=\varepsilon_{N, r m s}^{2}+\left[\frac{e \Psi_{0}}{2 \pi m_{e} c}\right]^{2},
$$

where $P_{\theta}$ is the canonical angular momentum of the beam, $\Psi_{0}$ is the flux through the cathode, $e$ is the charge of the electron, $m_{e}$ is the electron's rest mass, and $c$ is the speed of light. If the canonical angular momentum of the beam is measured, its contribution to the beam's envelope can be taken into account and the actual beam emittance determined. The purpose of the simulations was to determine if a non-zero cathode flux would increase the actual emittance in addition to producing a canonical angular momentum.

Two simulations using $\pm 30 \%$ variation in bucking coil field strength were performed. This variation is equivalent to changing the axial field on the cathode by $\pm 22 \mathrm{G}$ or an equivalent effective emittance of approximately $190 \mathrm{~cm}-\mathrm{mr}$. Results are shown in Figure 16. There appeared to be no significant change in emittance for variations in the bucking coil field strength of $\pm 30 \%$. The variation between the three simulations is due to a mismatch in the transport focusing rather than an actual emittance increase. 


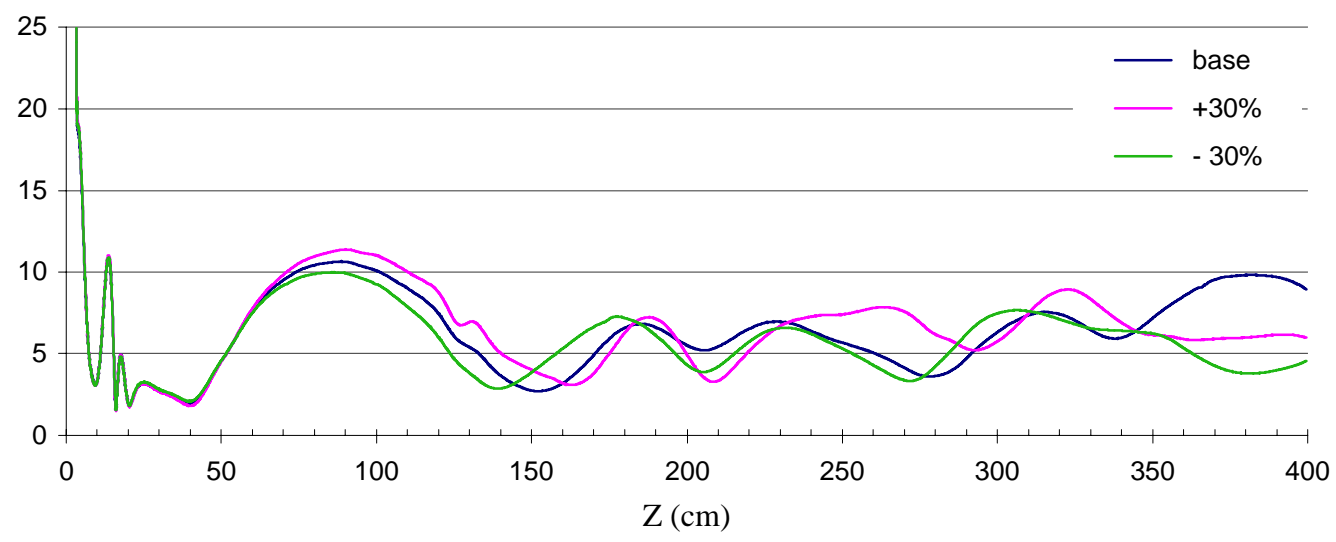

Figure 16. Emittance as a function of axial position for different Bucking Coil currents.

\section{Shroud Emission and Arcs}

The aluminum cathode shroud is anodized to suppress emissions to well below the Child-Langmuir Law limit and for practical considerations we would expect that shroud emission could be ignored. However, small imperfections in the anodized surface or defects that develop over time could lead to small areas where the emission may be higher than expected. ESTAT was used to calculate field stresses around the cathode shroud and then TRAK was used to estimate the level of emission for different surface preparations. Details of these estimates can be found in Reference 3. Figure 17 illustrates the electron orbits including shroud emission, limited to $2 \%$ of the Child-Langmuir Law, and the normal emission from the velvet cathode.

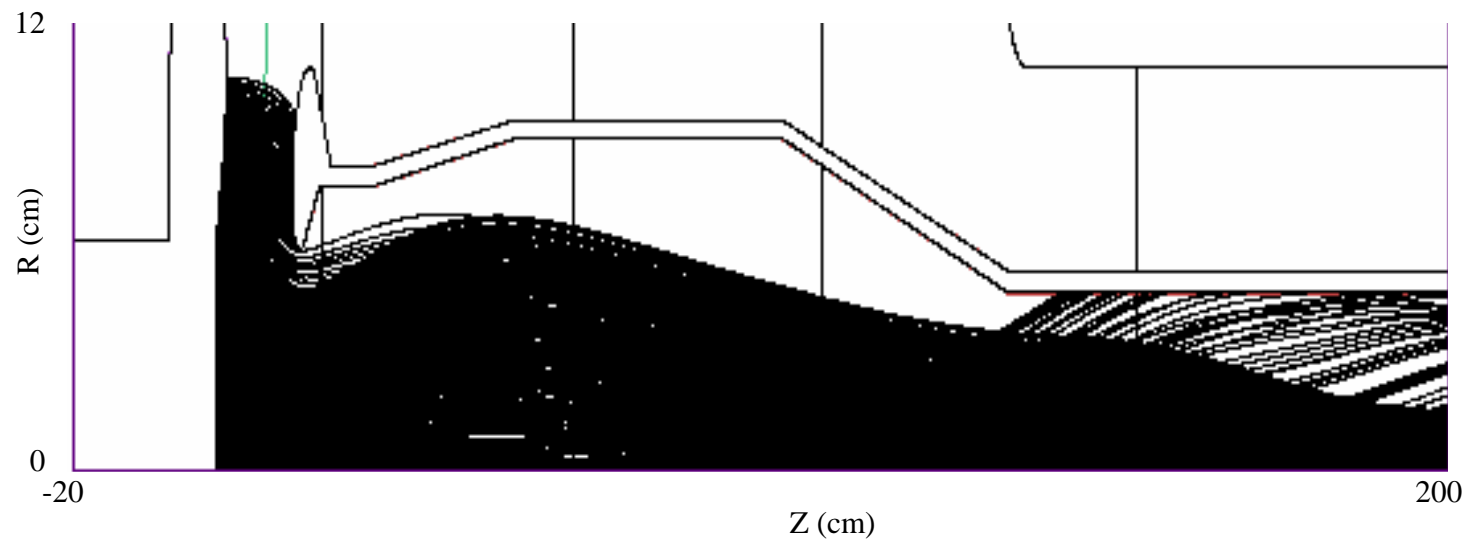

Figure 17. Simulated electron orbits are shown emitted from both the cathode and shroud.

The orbits in Figure 17 can be compared to those originating solely from the cathode illustrated in Figures 6 and 9. A total current of 246 A is emitted from the shroud. Approximately $100 \mathrm{~A}$ is intercepted on the anode tip and the remaining shroud orbits enter the main beam. The beam radius along the injector is shown in Figure 18. The shroud-emitted current has little effect on the rms radius in the A-K gap and even less after the gap as nearly half of this current is stopped at the anode tip. The maximum radius orbits reveal the extent of emission from the shroud and location of the anode tip. The shroud-emitted current that is not stopped at the anode tip enters the body of the beam, i.e. the cathode emitted current, around $50 \mathrm{~cm}$. At approximately $130 \mathrm{~cm}$ the orbits of the shroud-emitted current begin to separate from the main beam and actually intercept the $4.2 \mathrm{~cm}$ radius wall. 


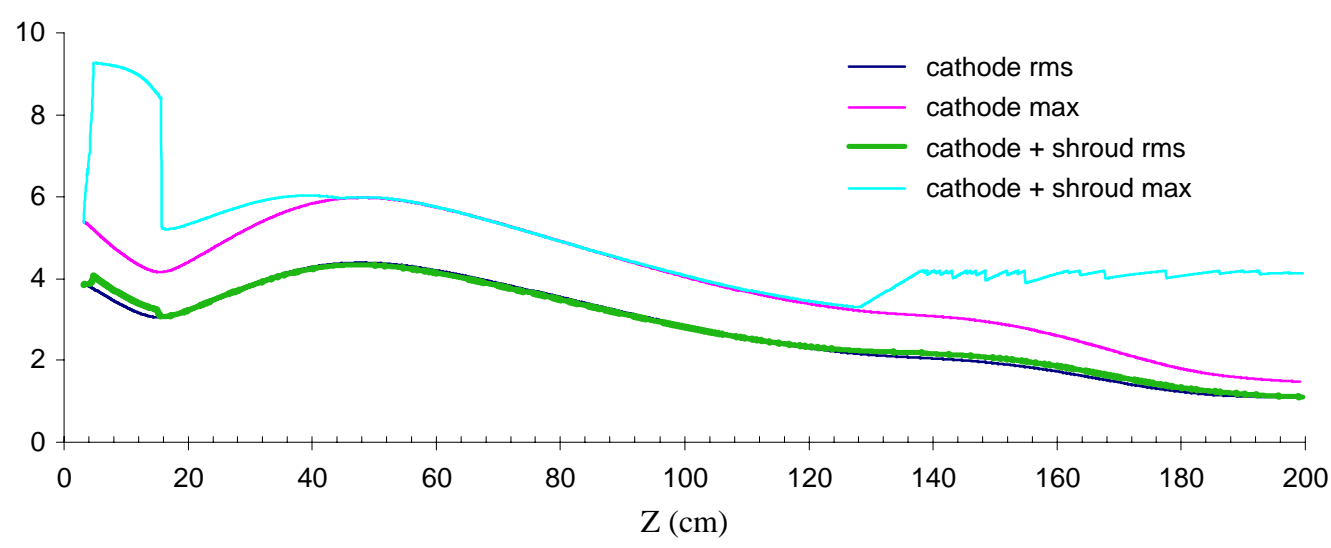

Figure 18. Maximum and rms radii for orbits from both the cathode and cathode-shroud are shown.

The emittance change due to the shroud-emitted current is illustrated in Figure 19. As expected, the significantly different trajectories of the shroud-emitted current compared to the cathode current results in a large increase in emittance. The decrease in emittance after $140 \mathrm{~cm}$ was not anticipated, but is obviously the result of preferential scrapping off the shroud-emitted current with respect to cathode emissions. The injector acts as an emittance selector!

Non-axially symmetric emission from the shroud is also possible. Precursors to electrical breakdowns across the diode could be small areas of plasma forming on the shroud. Assuming a plasma velocity of 2 $\mathrm{cm}$ per microsecond, in $50 \mathrm{~ns}$ the initial point of plasma formation would expand to a radius of $1 \mathrm{~mm}$. Child-Langmuir Law limited emission would be expected from these small bubbles of plasma.

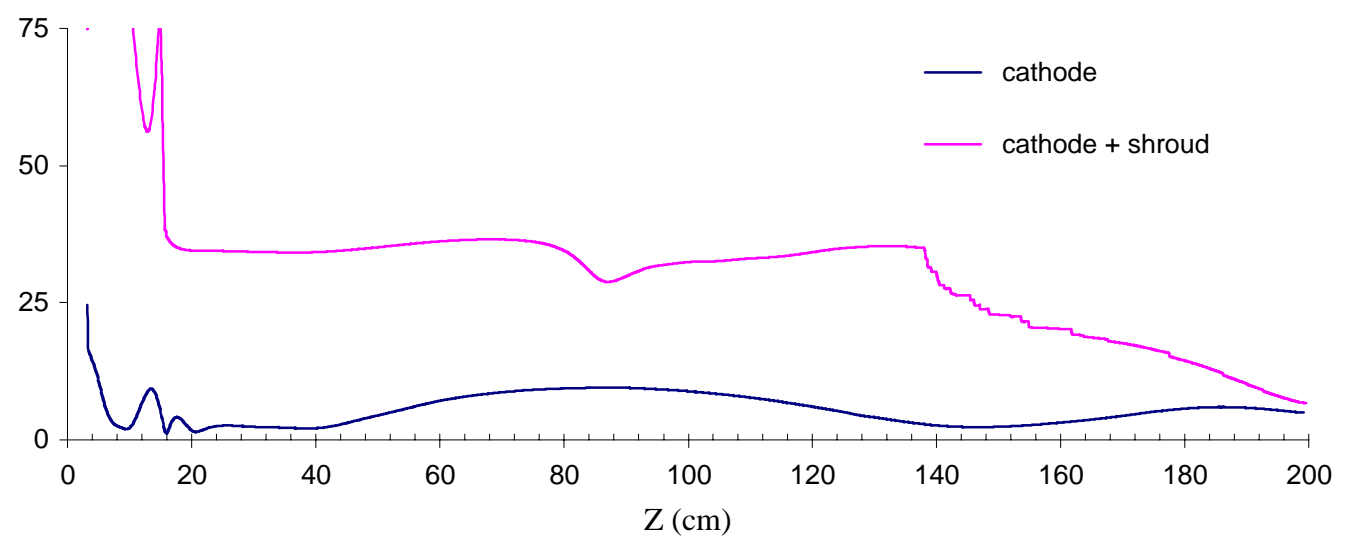

Figure 19. Emittance for emission from both the cathode and cathode-shroud are shown.

Images of the cathode in visible light taken with a gated, intensified camera detected a possible arc late in the pulse. Figure 20 shows images of the cathode taken at $\mathrm{FXR}^{27}$. The left image is a reference image taken with external lighting on the velvet. The image on the right was taken with a 10-ns gate approximately 70 ns after the start of the voltage pulse. This image has been scaled from 100 to 1,000 counts so that the glow from the plasma over the velvet is visible. Portions of the saturated region designated as the arc exceeded the 64,000 count of the camera. Refer to Figure 27 for a comparison to an image taken during the "flat top" portion of the pulse.

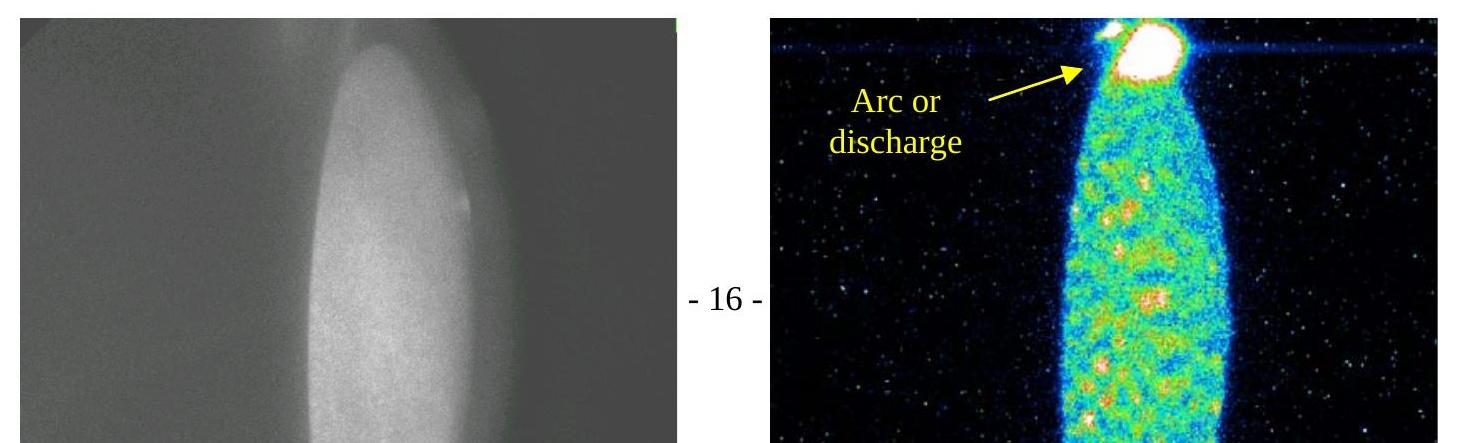


Camera image with external light.

$10 \mathrm{~ns}$ exposure about $70 \mathrm{~ns}$ after start of pulse.

Figure 20. Images of the FXR cathode showing a possible shroud arc.

Both OmniTrak and LSP were used to study the effect of emission from a small area of plasma on the surface of the cathode shroud. In OmniTrak a one-mm radius sphere was embedded in the cathode shroud at the location of the peak electric field to simulate such a plasma bubble. Figure 21 illustrates the location of the plasma bubble and the emission orbits. The potential of the bubble was the same as the shroud, -1.26 MV. The current from the plasma bubble was 54 amperes while the main beam was 3,200 amperes.

A plot of emittance along the Z-axis is given in Figure 22. The orbits from the shroud were not included in calculating the emittance. The large increased in emittance was not expected for the relative small shroud emission. However, the variation in transverse magnetic field across the beam was approximately 2 Gauss due to the 54 amperes of shroud emission. A 2-Gauss field represents a significant fraction of the total magnetic focusing field on the beam in the area of the diode. Additionally, this non-uniform deflecting field affects the orbits while their energy is still low.

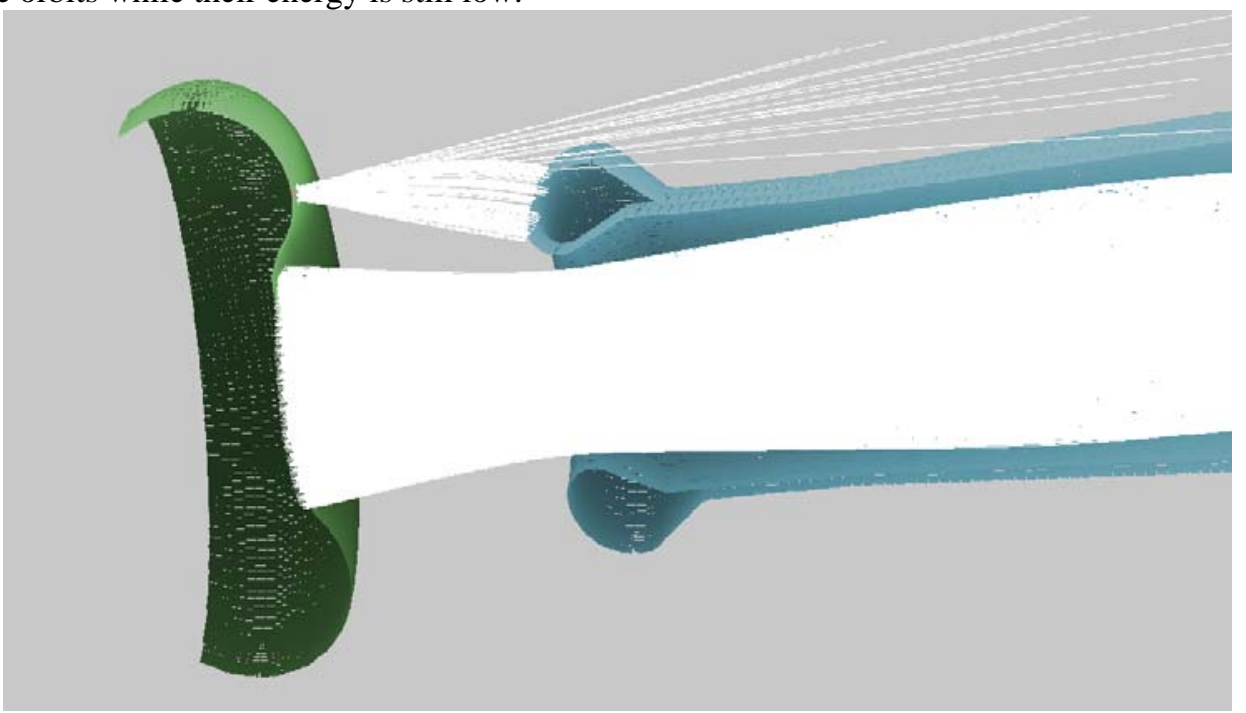

Figure 21. Three-dimensional depiction of emission from a small plasma bubble. 


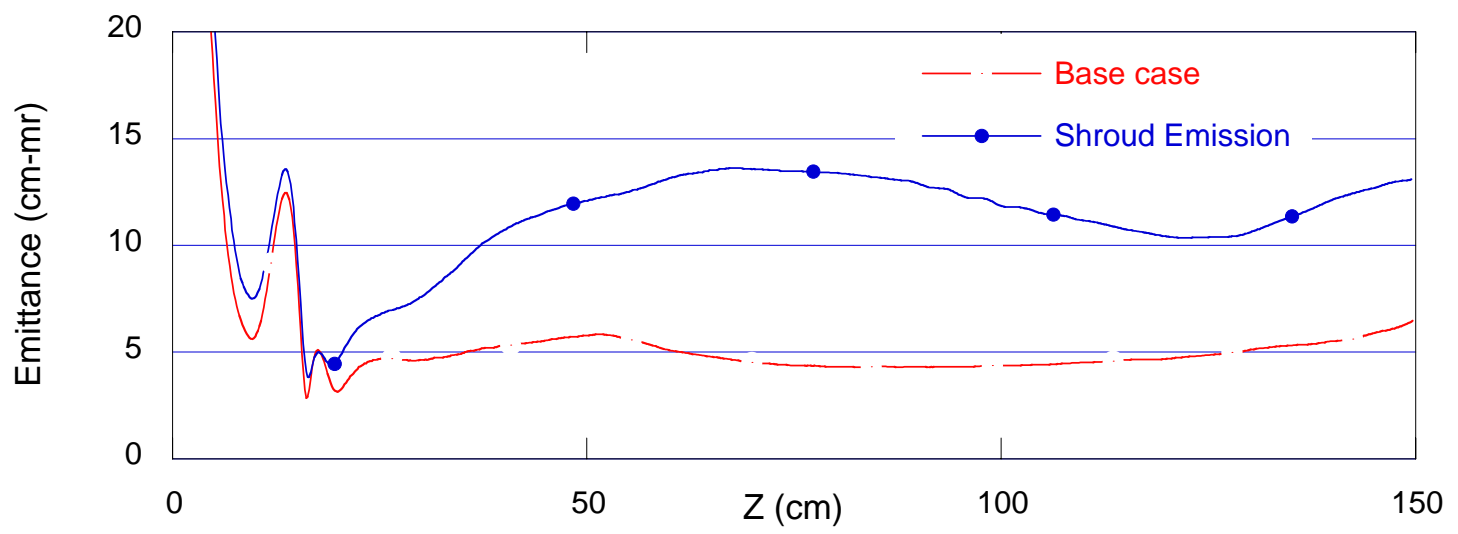

Figure 22. Comparison of emittance for the primary beam with and without emission from the shroud.

LSP simulation used a 3D cylindrical mesh with16 uniformly space $\theta$ nodes. Figure 23 illustrates the simulated arc emission area location on the shroud. Since there were only $16 \theta$ nodes, the arc emission region was not circular and had a total emission area of $1.5 \mathrm{~cm}^{2}$. The electrons emitted from this region produced a current of $700 \mathrm{~A}$ compared to 3,300 A for the velvet region. Similar to the OmniTrak simulation, all of the shroud emission was intercepted by the anode tip and did not enter the main beam.
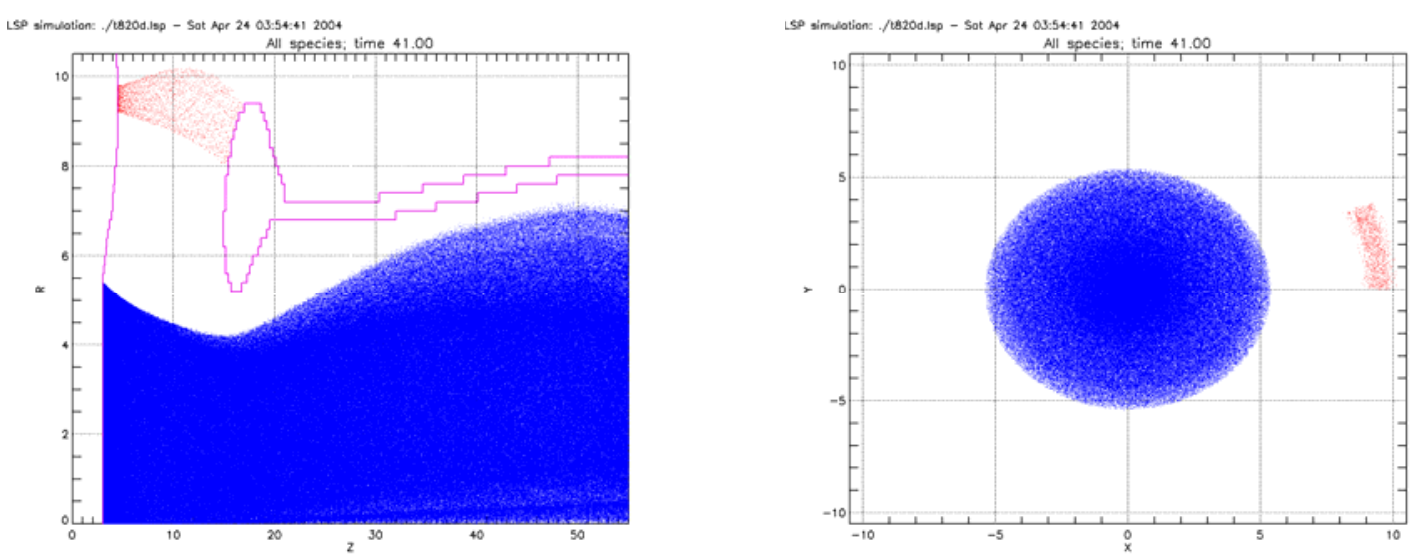

Figure 23. Illustrations of the shroud emission electron orbits (red) and the velvet electrons (blue).

The LSP calculated emittance for the "flat top" portion of the pulse (41 ns) as a function of axial position is shown in Figure 24.
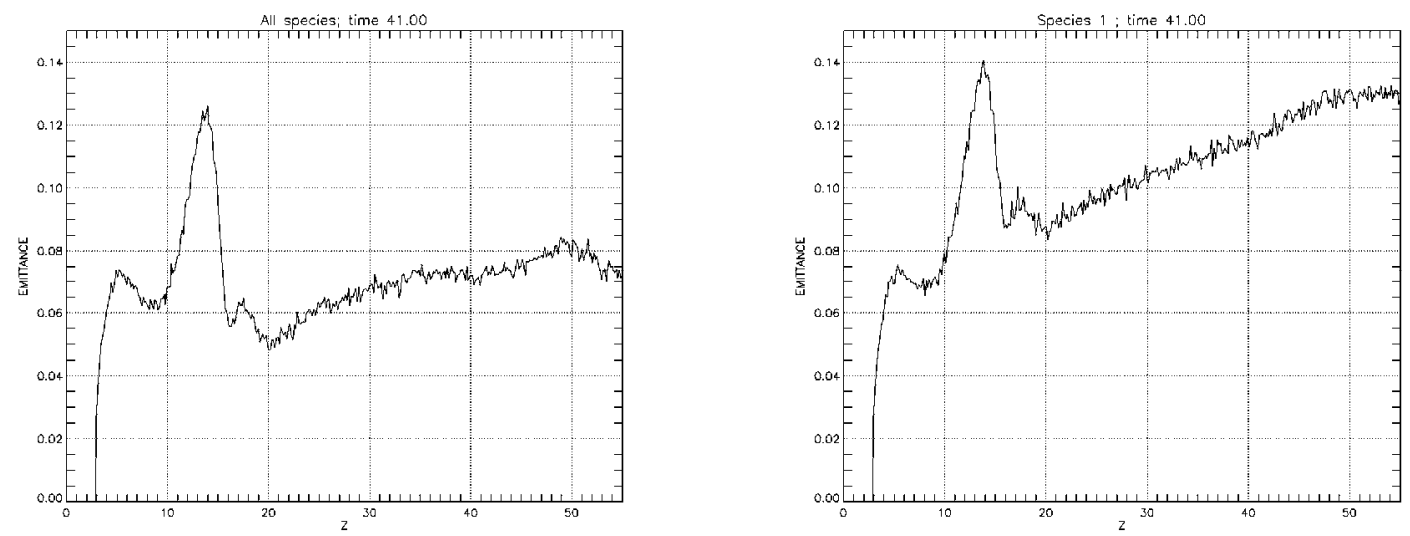

Figure 24. Lapostelle normalized emittance at $\mathrm{t}=41 \mathrm{~ns}$ with/without shroud emission. 
OmniTrak indicated a doubling of emittance with a much smaller shroud current compared to LSP's 40\% increase. This may be due to the more localized emission source in OmniTrak effecting one portion of the electron orbits strongly while the less localized LSP source had a smaller effect on orbits. The OmniTrak source had over three times the current density as LSP. The TRAK results for an axially symmetric shroud emission supports this conjecture.

\section{Non-Uniform Emissions}

The emission surface obviously has an important influence on beam quality. Microscopic variations are a probable cause of higher than anticipated "thermal" emittance ${ }^{28}$. Non-uniform discoloration of FXR's velvet cathode surface with usage has led to speculation of non-uniform emission across the cathode.

TRAK was used for the initial study of non-uniform emission. The emission surface was divided into ten concentric bands of equal surface area. The emission from individual rings was then limited for different simulations. Figure 25 shows two examples. For the top example, emission was suppressed for the center of the velvet. This simulation was motivated by the large discoloration noted on the velvet after usage. In the bottom example the emission was suppressed for a band centered about a radius of $2.1 \mathrm{~cm}$. The beam emittance for the various rings simulations is given in Figure 26. Full indicates that the entire cathode surface was emitting while center (edge) indicate no emission from the center (edge) of the cathode. The numbers refer to non-emitting bands where lower numbers are nearer the center. Several interesting features from this series of simulations were noted. Although $10 \%$ of the surface area had suppressed (no) emission, the total emitted current decreased by only approximately $3 \%$. This was due to increased current density from the area adjacent to the non-emitting area. The orbits in Figure 25 diverge significantly from the uniform case, but the rms radius of the total beam remained essentially constant. The greatest increase in emittance was for the center portion of the velvet to be non-emitting. At an axial location of $155 \mathrm{~cm}$, this simulation indicated current loss/wall intercept of the orbits that originated near the non-emitting area. Their lost improved the emittance similar to other collimating techniques for improving emittance and to the situation for axially symmetric shroud emission described in the previous section. Non-emission from the outer band had very little impact on emittance.

The large increase of emittance when the center of the cathode was non-emitting motivated an experiment to measure the plasma light from the FXR velvet cathode. Sample images from this experiment are shown in Figure 27. Refer to Figure 20 for orientation. The visible light, presumably associated with the plasma generated from the velvet flash over, shows considerable texture. Similar images of plasma light from velvet cathodes have been published ${ }^{29,30}$, and the FXR images are not unusual.

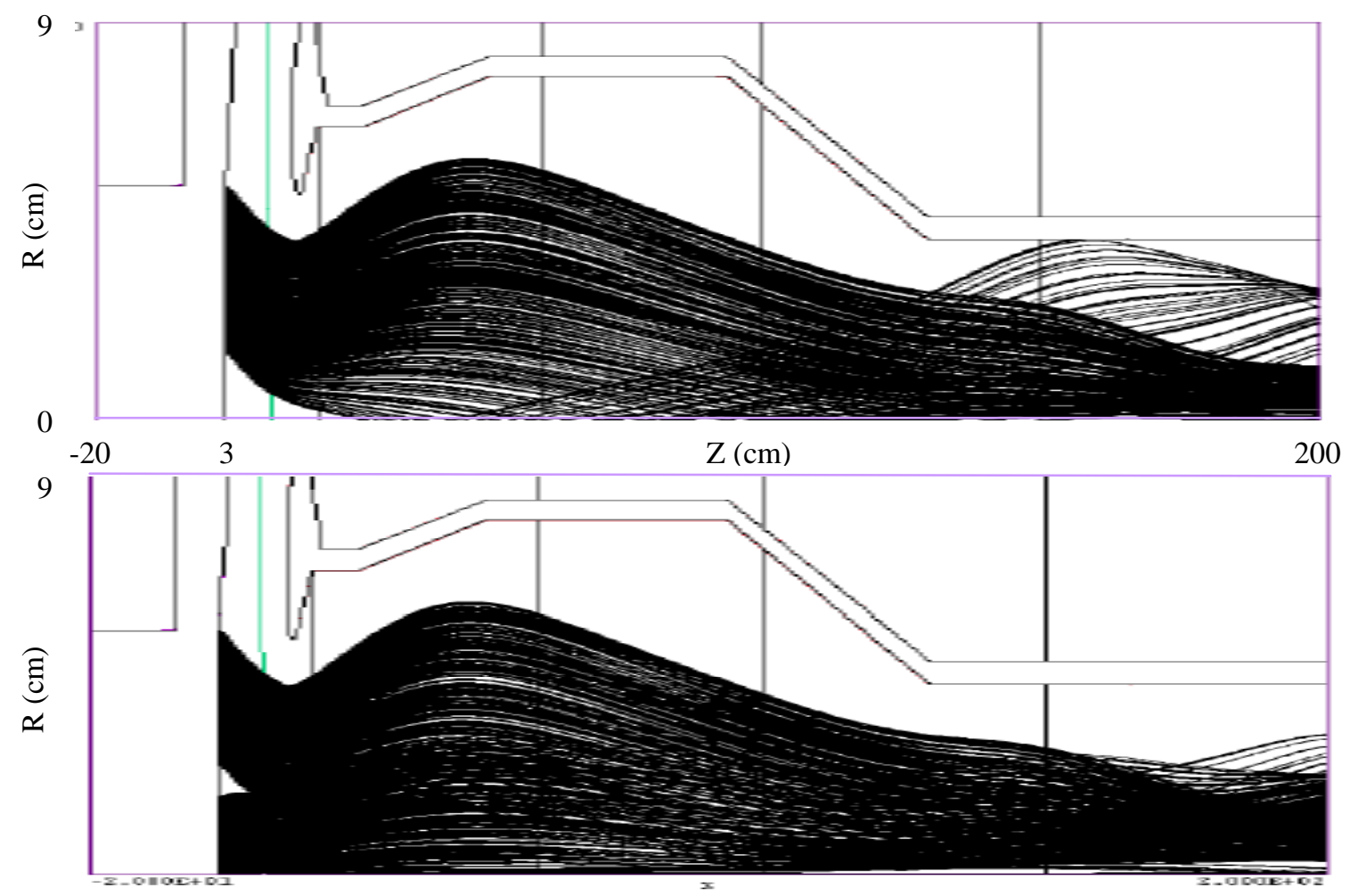


Figure 25. Electron orbits are shown for simulated no emission from two different areas of the cathode.

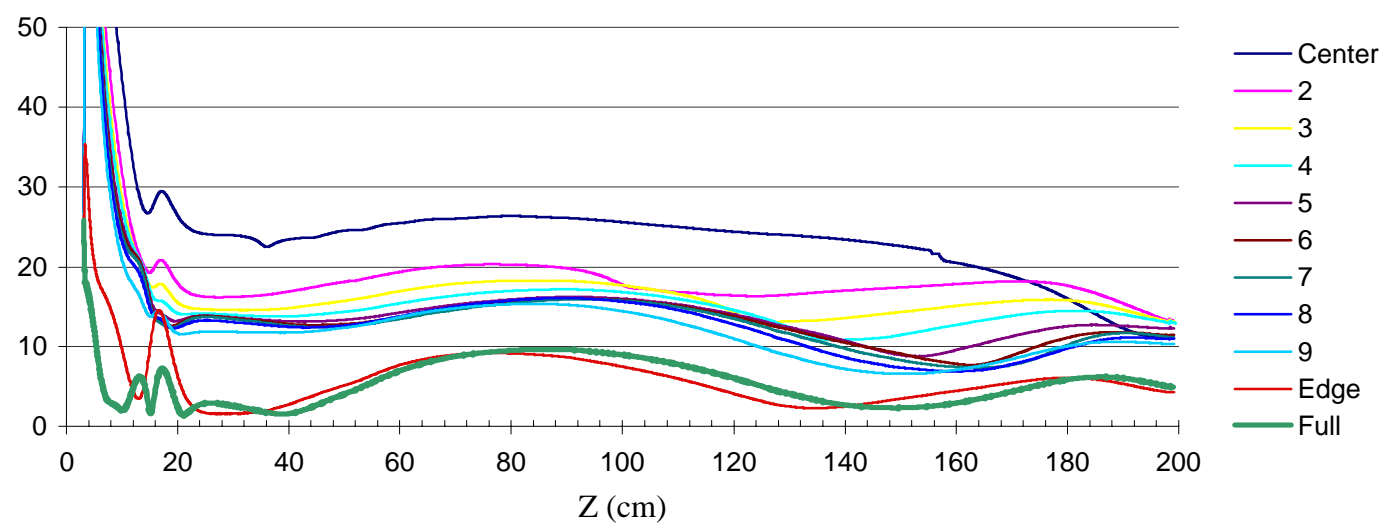

Figure 26. Emittance is shown as a function of axial position for different bands of non-emission.

The resolution or grid size needed to simulate the three-dimensional pattern of emission centers shown in Figure 27 was far beyond our simulation capabilities. In an attempt to understand the importance of nonuniform, non-axial symmetric emission from the velvet surface we simulated relatively large non-emitting areas on the cathode surface with OmniTrak and LSP.

Three OmniTrak simulations were performed with current emission suppressed over different portions of the emitting surface. All three simulations suppressed emission over equal areas, approximately $3.4 \%$ of the total emitting area, of the cathode surface. The difference was that the non-emitting area was divided between one, two, or three localized spots. Figure 28 shows the distribution of orbits immediately in front of the cathode for the simulations where the non-emitting area is divided over two (left) or three circular areas. The orbits adjacent to the non-emitting disks had higher current than the average due to the absent space charge of non-emitted orbits. Emittance as a function of axial location is shown in Figure 29 for the three cases.
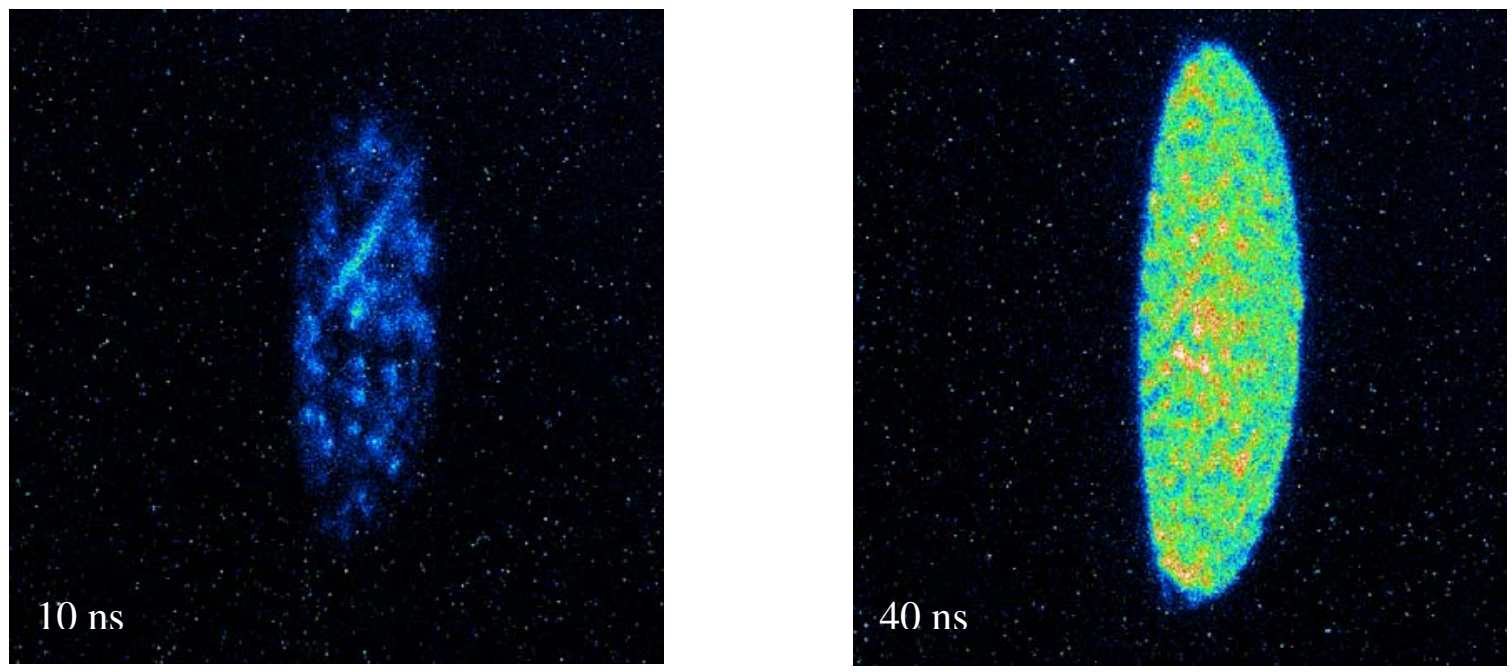

Figure 27. Images of velvet cathode during emission are shown during rise time (left) and "flat top". 

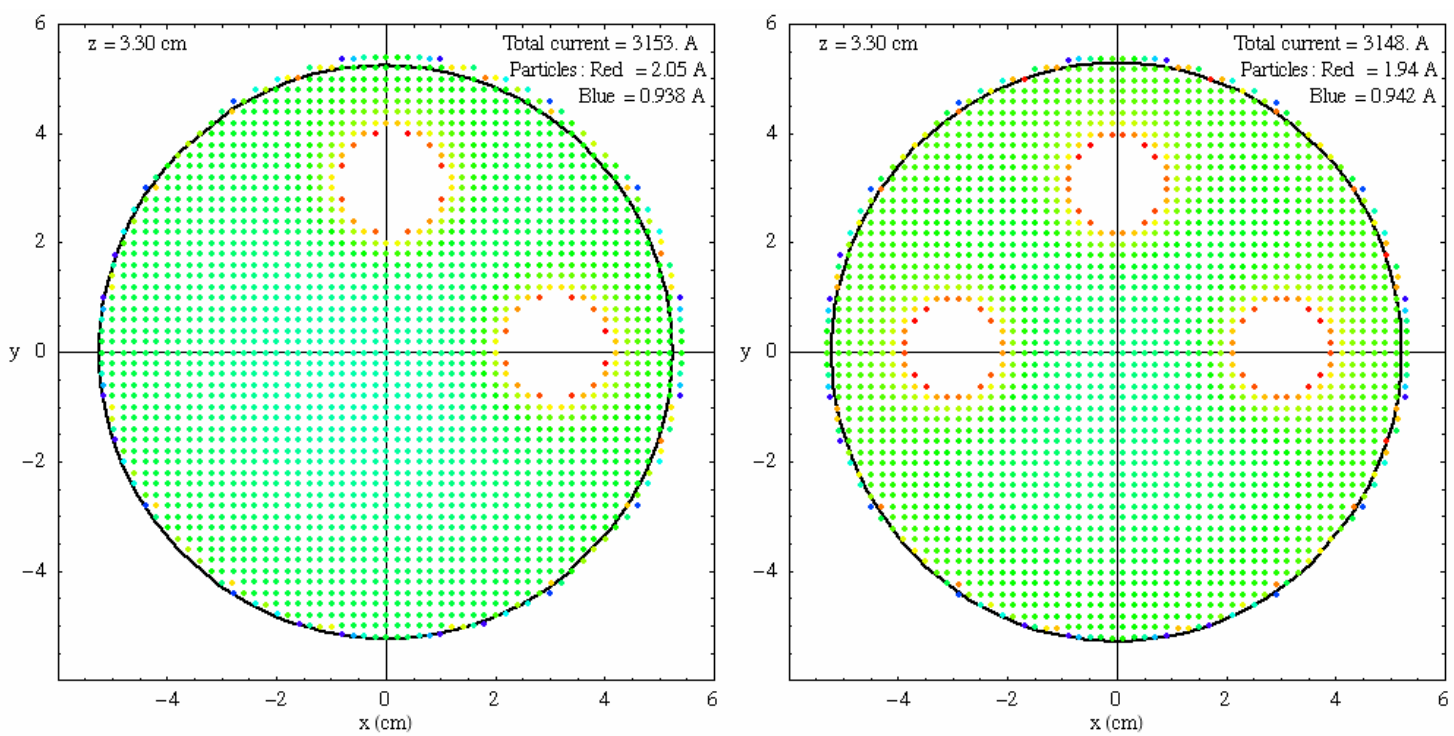

Figure 28. X-Y plots of orbits immediately in front of the cathode for two different simulations.

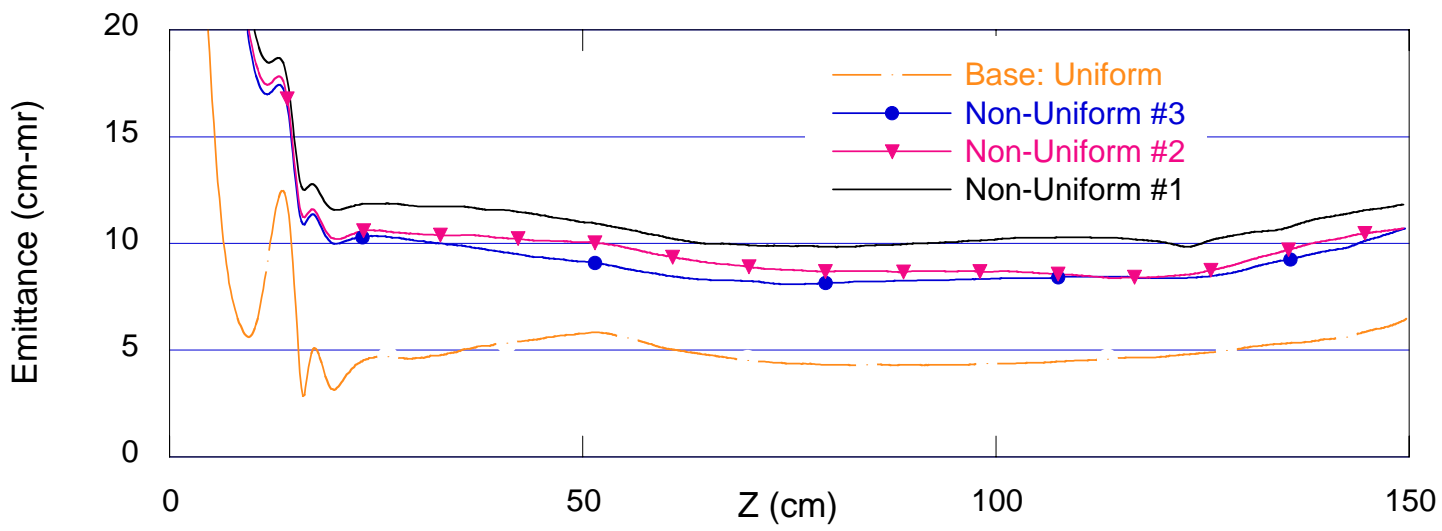

Figure 29. Emittance for different types of non-emitting surfaces.

A single non-emitting surface increased the emittance more than multiple non-emitting surfaces enclosing an equal area. If the total non-emitting area remains constant, dividing that area over more surfaces should lower the emittance. In the limit of infinite surfaces spread randomly over the cathode, emittance for uniform emission should be realized.

The LSP simulation used a small, non-emitting area $\left(1.37 \mathrm{~cm}^{2}\right.$ or somewhat less than one half of the 3.14 $\mathrm{cm}^{2}$ used in the OmnitTrak simulations) placed in the emission region as shown in Figure 30. The emittance as a function of time at an axial position of $50 \mathrm{~cm}$ is given in Figure 31. 

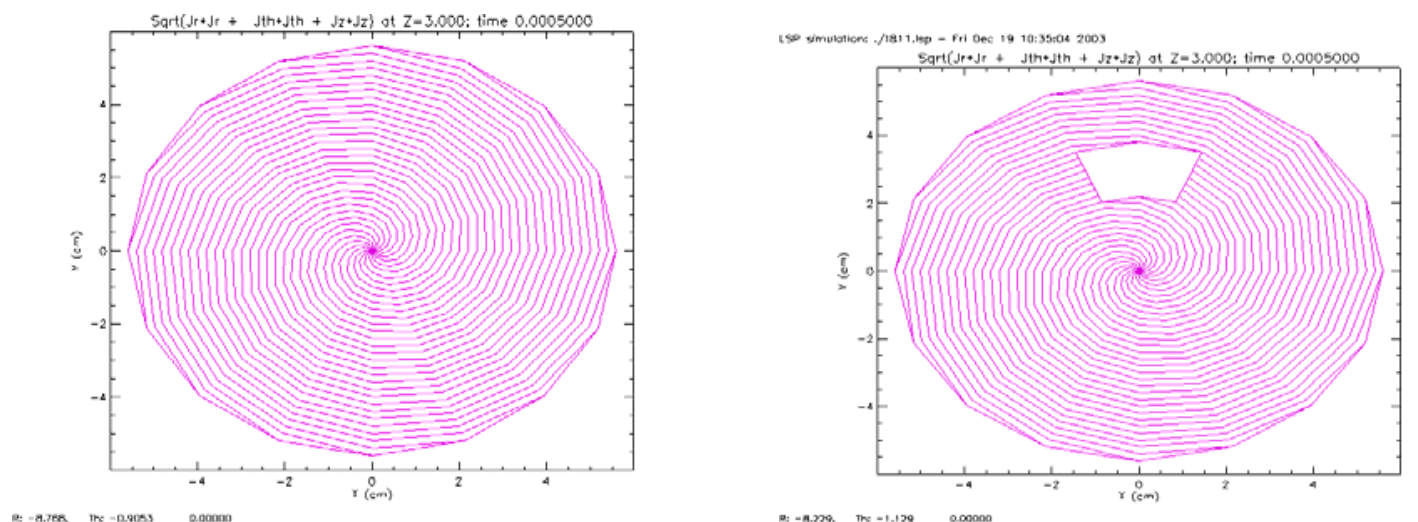

Figure 30. LSP geometry used for the non-uniform emission study.
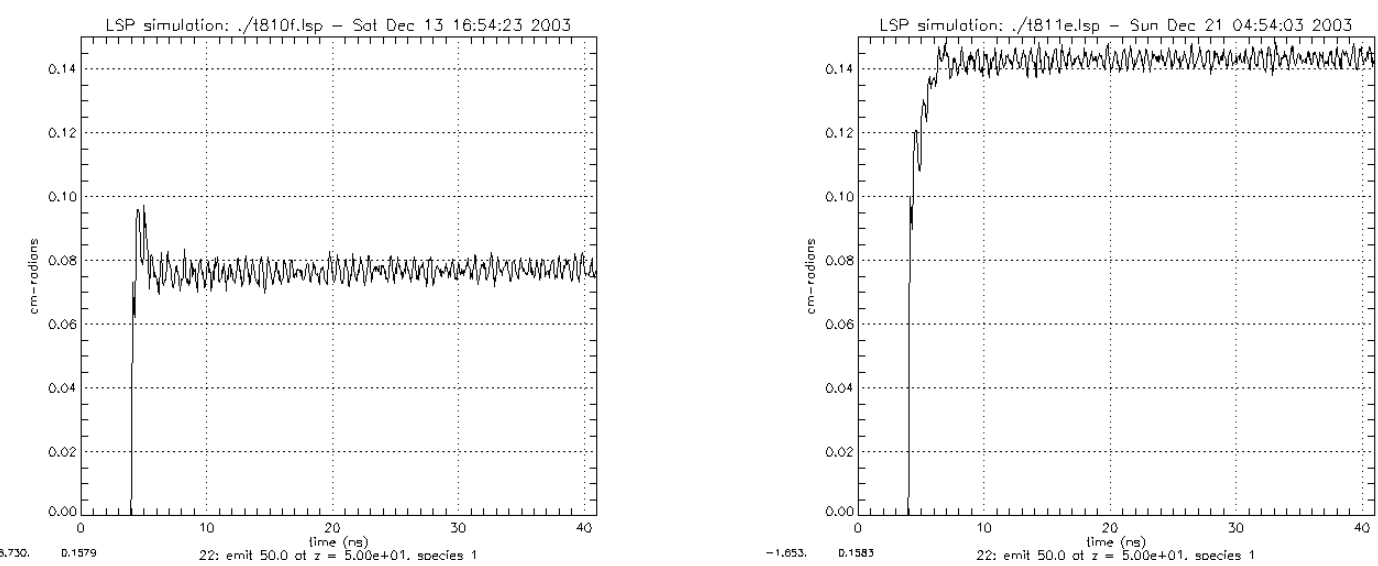

Figure 31. Lapostelle normalized emittance at $\mathrm{z}=50 \mathrm{~cm}$ for uniform (left), non-uniform (right) emission.

\section{Conducting Foil Effect on Emittance}

A technique for measuring a space charge dominated beam is to pass it through an aperture, or array of apertures referred to as a pepperpot, that limits the transmitted current to a level that the beam becomes emittance dominated. The expansion of this low current beam, or beamlet, can then be measured yielding the emittance. A major assumption is that the emittance of the beamlet is representative of the beam prior to passing through the aperture. Many issues need to be addressed in a successful measurement, e.g. scattered electrons from the aperture, vignetting, transmission of electrons through the blocking foil around the aperture, etc. Most of these issues can be made acceptably small through careful design of the hardware. A more awkward assumption is that the beam at the entrance of the aperture is representative of the beam when no aperture/foil is present. This assumption is necessarily false since the aperture foil, or mask, distorts the electric field in the vicinity of the mask and changes some of the properties of the beam. The foils are normally conducting and grounded with respect to the local beam pipe to avoid problems with accumulating charge.

Track and LSP were used to simulate the influence of a thin metal, grounded foil on the beam emittance. The LSP simulation was more ambitious with the simulated foil placed at an axial position of $414.4 \mathrm{~cm}$, the location where the FXR pepperpot measurements are typically taken. Plots of the electron orbits for steady state conditions are shown in Figure 32 for simulations with and without the conducting foil. The effect of the foil on the beam envelope prior to the foil is very small. For the simulation, the foil is range thin for the beam and foil focusing is obvious after passage. The important finding is that the emittance of the beam increases by 50\% immediately in front of the foil over the no foil case as shown in Figure 33. A very carefully performed measurement of the beam emittance at the pepper pot aperture array would yield an emittance significantly greater than that of the undisturbed beam. 

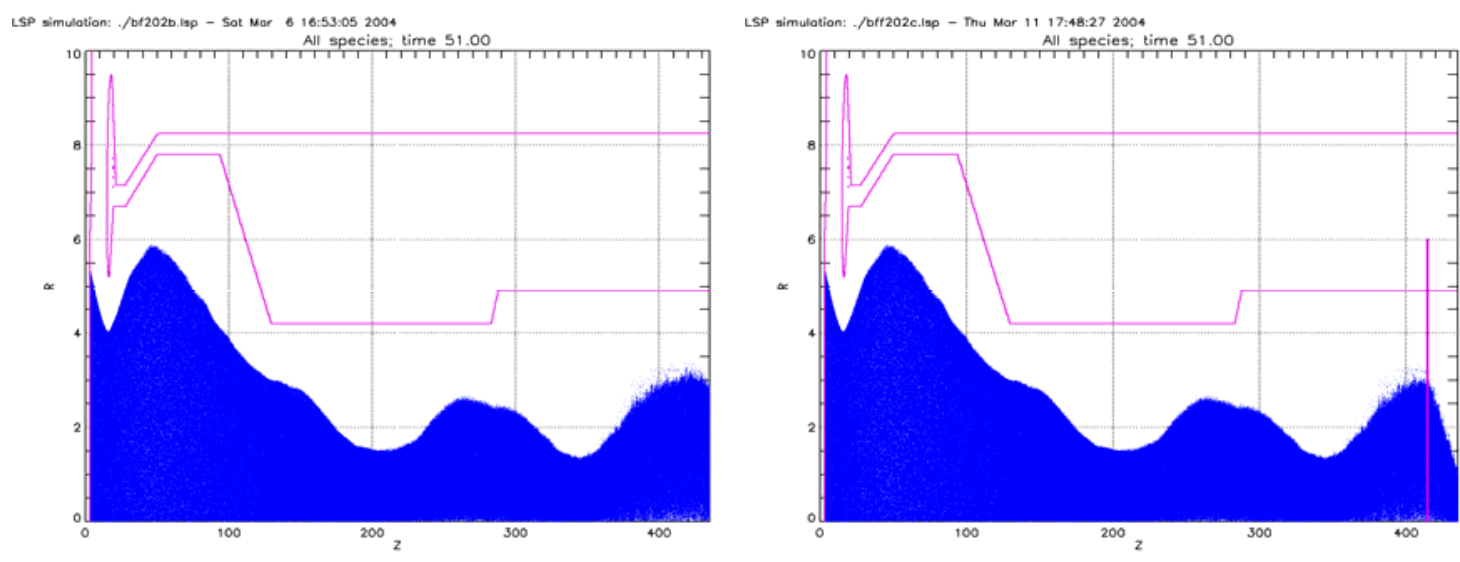

Figure 32. Electron orbits with and without a conducting foil at $\mathrm{z}=414.4 \mathrm{~cm}$.
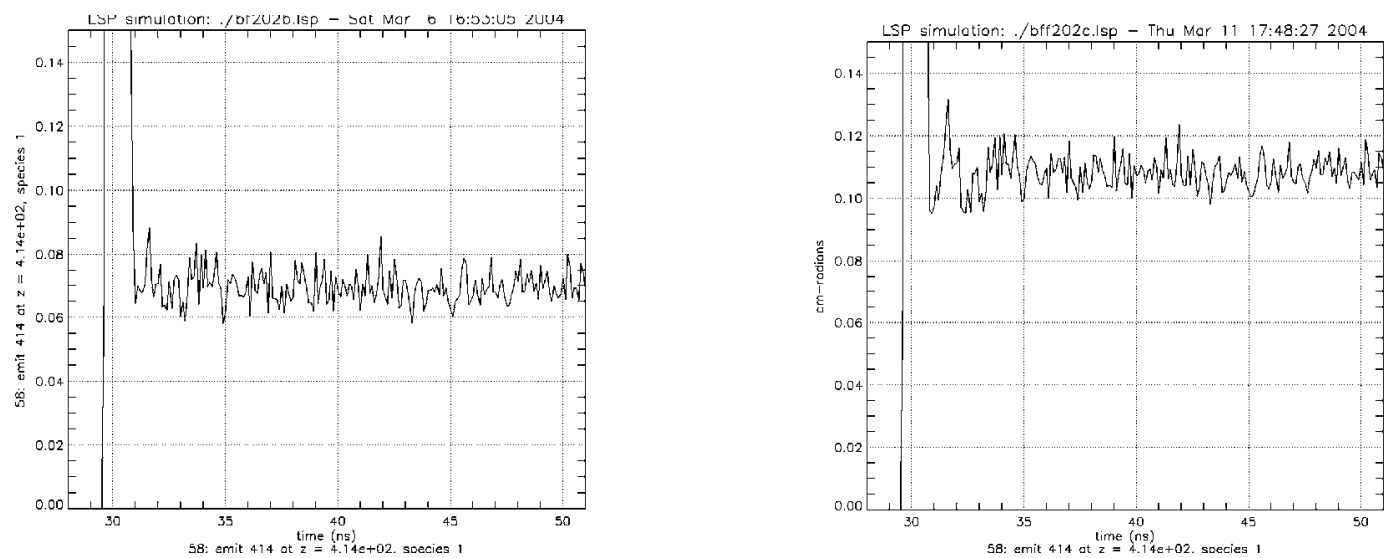

Figure 33. Beam emittance versus time at $\mathrm{z}=414 \mathrm{~cm}$ with/without a grounded, conducting foil.

TRAK simulations could not be extended to $414 \mathrm{~cm}$ to simulate the FXR emittance measurements due to numerical instability. For purposes of studying the effect of conducting foils, The TRAK simulation covered an axial distance of $200 \mathrm{~cm}$ with the foil inserted at $180 \mathrm{~cm}$ and included varying the thickness of the foil. TRAK simulation results were similar to LSP with respect to the beam envelope. However, the change in emittance was strikingly different. Calculated emittance is shown in Figure 34. Unlike the LSP results at $414 \mathrm{~cm}$, TRAK found a decrease in emittance by a factor of two for the foil at $180 \mathrm{~cm}$. A pepper pot measurement performed at this location would measure an emittance for the beam half as large as for the standard beam.

The simulations indicated that the beam envelope at the plane of the foil did not change significantly with the presence of the foil. Thus, emittance based on beam envelope, i.e. radius, measurements should be more straightforward than pepperpot measurements. The difficulty remains in separating the effect of space charge from emittance on the beam envelop. 


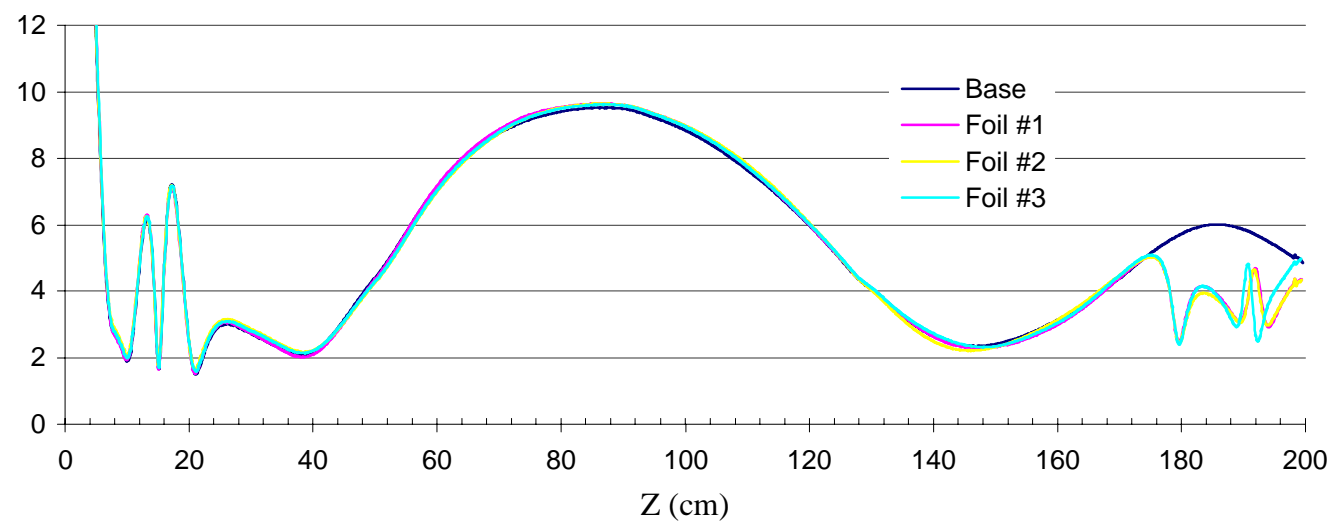

Figure 34. Emittance as function of axial position with/without a grounded, conducting foil at $180 \mathrm{~cm}$.

\section{Conclusions}

There are there several conclusions from the parameter study. Foremost is that the present diode design should produce a beam with much lower emittance than what has been measured. To resolve this discrepancy approximately equal time was spent benchmarking the three codes against each other and simulating the effects of perturbations to the nominal injector design. In other words we wanted to ensure that the simulations were believable and, if so, where the fault rested in the injector.

The three codes agreed on total emitted current to $\pm 3 \%$ with the LSP 2-D and 3-D results at the average of the TRAK and OmniTrak values. All three codes agreed on the beam envelope for approximately the first $150 \mathrm{~cm}$ of the injector, the limit for an accurate OmniTrak simulation, and LSP and TRAK agreed out to approximately $400 \mathrm{~cm}$. Agreement on the beam envelope does not necessarily indicate agreement on beam emittance since the dominant influence on the envelope at this current, energy, and nominal radius is space charge. Referring to Figure 11, the codes all show different behavior for the emittance as a function of axial location. At $80 \mathrm{~cm}$ the highest predicted emittance is a factor of three greater than the lowest value. However, this is an absolute difference of $30 \mathrm{~cm}-\mathrm{mr}$ (normalized) compared to a difference of $160 \mathrm{~cm}-\mathrm{mr}$ (normalized) between the average simulated $\left(\varepsilon_{N, r m s}=36 \mathrm{~cm}-\mathrm{mr}\right)$ and measured $\left(\varepsilon_{N, \mathrm{rms}}=193 \mathrm{~cm}-\mathrm{mr}\right)$ values. The agreement between TRAK and LSP improves as the magnetic field becomes more uniform further along the injector. The conclusion of the three code simulations is that the FXR injector should have much lower emittance.

The next conclusion is that the present diode design is robust with respect to perturbations to the nominal design. Easily detected mechanical alignment/position errors and magnet errors did not lead to appreciable increase in the simulated emittance. Refer to Table 3.

Since the codes are consistent but do not agree with measurements, there must be some physics that is not being modeled correctly. The only significant area where the three codes omitted detailed physics in the modeling was with regards to electron emission. The assumption was made that the velvet, emitting surface could be treated as a uniform, infinite source of electrons with emission limited by the Child-Langmuir Law. Thermal, i.e. intrinsic, emission was an input parameter to the codes. Initial simulations also assumed no emission from the cathode shroud. Attempts to understand and correct for these modeling deficiencies led to a number of interesting observations, both in simulations and experiments.

1) Simulations of uniform emission from the anodized aluminum shroud at a relatively high $2 \%$ of the Child-Langmuir Law limit indicate a halo of electrons would be produced. However, those electrons would be preferentially lost on the walls of the injector within $200 \mathrm{~cm}$ and have an insignificant impact on emittance at the exit of the injector. 
2) Thermal emittance was not a significant contributor to the total emittance.

3) A large (10\% of total cathode area) non-emitting area in the center of the cathode could double the simulated emittance. The effect on emittance was enhanced if the non-emitting area was not concentric with the cathode, i.e. if axial symmetry was broken. These findings led to experiments that imaged the plasma light over the velvet surface. The images did not indicate large, low emission regions, but did show a mottled appearance that was beyond our capability to model.

4) The plasma light images captured a late pulse time "arc-like" discharge on the cathode shroud. Simulations of a plasma bubble located on a high field stress region of the cathode with ChildLangmuir Law limited emission found a doubling of the beam emittance. Although intriguing, the lateness of the arc with respect to the beam pulse and random occurrence should eliminate this phenomenon as a cause of the consistently high injector emittance.

A different approach was to question the emittance measurement at the exit of the FXR injector. The measurement used the pepperpot technique where the majority of the beam current is stopped on a range thick, conducting foil with an array of small apertures. The "beamlets" of current that pass through the foil are emittance dominated and the expansion of their orbits can be related to the incoming beam emittance at the foil. LSP simulations of the beam intercepting a conducting foil at the location used for the measurement showed a doubling of emittance at the foil compared to no foil. Thus, the measured emittance would be twice as large as the actual emittance.

These conclusions lead to a possible explanation for the larger than expected injector emittance. Nonuniform emission from the cathode is responsible for doubling the expected emittance. The pepperpot aperture foil increases the beam emittance by another factor of two. The combination of the two effects would be sufficient to explain the discrepancy between predicted and measured emittance within reasonable uncertainty of the measurements. It would also indicate that the more serious emittance growth is in the accelerator.

Two recommendations are offered. The first is a relatively easy experiment to address the explanation given in the previous paragraph for the larger than expected emittance. There are two necessary requirements for the experiment to be successful. The first is that inserting a foil into the beam has negligible effect on the beam envelope at the point of insertion. This was shown to be true in simulations. Refer to the previous section Conducting Foil Effect on Emittance.

The second involves the relative contributions of emittance and space charge to the beam envelope. The envelope equation ${ }^{31}$ for a beam in vacuum with the assumptions of equilibrium, i.e. constant radius, and no scattering can be expressed as:

$$
\left(\frac{e B_{z}}{2 m_{e} c}\right)^{2} r^{4}-\frac{I_{b}}{\gamma \beta I_{a}} r^{2}-\varepsilon_{N, r m s}^{2}=0, \text { where } I_{a}=\frac{I_{A}}{\gamma \beta}=17 \mathrm{kA} .
$$

We can define two quantities representing the equilibrium radius for the cases of zero beam current $\left(I_{b}=0\right)$ and zero emittance $\left(\varepsilon_{N, \text { rms }}=0\right)$ :

$$
r_{e}=\left(\frac{2 m_{e} c}{e B_{z}} \varepsilon_{N, r m s}\right)^{1 / 2} \text { and } r_{i}=\frac{2 m_{e} c}{e B_{z}}\left(\frac{I_{b}}{\gamma \beta I_{a}}\right)^{1 / 2} .
$$

Substituting the expressions of Equation 2 into Equation 1: $r^{4}-r_{i}^{2} r^{2}-r_{e}^{4}=0$.

The Ratio value listed in Table 4 is defined as: Ratio $\equiv \frac{\gamma \beta I_{a} \varepsilon_{N, r m s}^{2}}{I_{b} r^{2}}$.

Table 4 was generated with Equations 2, 3, and 4 for values of $I_{b}=3 \mathrm{kA}$ and $\gamma \beta=5$ (2.1 MeV beam). The information in Table 4 indicates the relative importance of space charge compared to emittance on the beam radius. For an emittance value of $40 \mathrm{~cm}-\mathrm{mr}$, representative of the simulation results, the beam envelope is totally dominated by space charge up to the highest magnetic field (1.2 kG) and smallest radius 
$(0.55 \mathrm{~cm})$ for a practical imaging foil experiment on the FXR injector. The beam is still space charge dominated at an emittance of $100 \mathrm{~cm}-\mathrm{mr}$, i.e. one half the measured value. However, the emittance contribution to the envelope is significant once the magnetic field is increased to one kilogauss. If the emittance is actually $200 \mathrm{~cm}-\mathrm{mr}$, the beam is emittance dominated for fields above $900 \mathrm{G}$.

Measurements of the beam envelope at the exit of the injector as a function of the strength of the focusing solenoids could be used to determine the emittance. Specifically, varying the strength of the I34 or J21 solenoid and imaging the beam at the J21 accelerator gap would provide sufficient information to confirm the pepperpot measurement. Note that for a sufficient variation of field strengths and radii, the beam energy and current could also be determined.

Table 4. Effect of increasing emittance on beam radius for different focusing field strengths.

\begin{tabular}{|c|c|c|c|c|c|}
\hline Emittance & $B_{Z}(\mathrm{kG})$ & $r_{e}(\mathrm{~cm})$ & $r_{i}(\mathrm{~cm})$ & $r(\mathrm{~cm})$ & Ratio (\%) \\
\hline \multirow{4}{*}{$\begin{array}{c}40 \mathrm{~cm}-m r \\
\text { (normalized } \\
\text { rms) }\end{array}$} & 0.4 & 0.58 & 1.60 & 1.61 & 2 \\
\cline { 2 - 6 } & 0.6 & 0.48 & 1.07 & 1.09 & 4 \\
\cline { 2 - 6 } & 0.8 & 0.41 & 0.80 & 0.83 & 7 \\
\cline { 2 - 6 } & 1.0 & 0.37 & 0.64 & 0.67 & 10 \\
\hline \multirow{3}{*}{$\begin{array}{c}100 \mathrm{~cm}-\mathrm{mr} \\
\text { (normalized } \\
\text { rms) }\end{array}$} & 1.2 & 0.34 & 0.53 & 0.57 & 14 \\
\cline { 2 - 6 } & 0.4 & 0.92 & 1.60 & 1.68 & 10 \\
\cline { 2 - 6 } & 0.6 & 0.75 & 1.07 & 1.17 & 21 \\
\cline { 2 - 6 } & 1.0 & 0.65 & 0.80 & 0.92 & 33 \\
\hline \multirow{3}{*}{$\begin{array}{c}200 \mathrm{~cm}-m \mathrm{r} \\
(\text { normalized } \\
\text { rms) }\end{array}$} & 1.2 & 0.58 & 0.64 & 0.78 & 47 \\
\cline { 2 - 6 } & 0.4 & 1.31 & 0.53 & 0.68 & 62 \\
\cline { 2 - 6 } & 0.8 & 1.07 & 1.60 & 1.85 & 33 \\
\cline { 2 - 6 } & 1.2 & 0.92 & 0.80 & 1.36 & 92 \\
\hline
\end{tabular}

After measuring the emittance with an envelope technique to verify the pepperpot results, the next recommendation is more difficult. A diode redesign and/or experiment are needed. The modest electrical field stress on the velvet surface may be the cause of non-uniform emission. Attempting to prove this will require changing the diode configuration to increase the field stress on the cathode, adjusting the focusing solenoids for the new configuration, and measuring the beam emittance. TRAK is sufficient for modeling the higher field stress design. However, as mentioned above, we do not presently have the capability to model the details of emission from the velvet surface. Actual measurements will be needed to see if the higher field stress cathode actually lowers the beam emittance

\section{References}

${ }^{1}$ Tim Houck, “Three Dimensional Parameter Study of the FXR Injector Diode,” FXR Note 2004-May-002, 10 May 2004

${ }^{2}$ Donald T. Blackfield, “Two and Three Dimensional Parameter Study of the FXR Injector Using the LSP Code,” FXR Note 2004-July-004, 8 July 2004

3 Jalal B. Javedani, “Two dimensional Parameter Study of the FXR Injector Using the TRAK Code,” FXR Note 2004July-005, 24 June 2004

${ }^{4}$ Arthur C. Paul, “FXR Injector Study,” UCRL-TR-203991, 31 January 2003

${ }^{5}$ E.P. Lee and R.K. Cooper, “General Envelope Equation for Cylindrically Symmetric Charged-Particle Beams,” Particle Accelerators, 7, 83 (1976)

${ }^{6}$ P.M. Lapostolle, "Possible Emittance Increase Through Filamentation due to Space Charge in Continuous Beams," IEEE Trans. Nucl. Sci. NS-18, No. 3, 1101 (1971) 
${ }^{7}$ E. Merle, et al., “Transport of the 1.92 - 3.1 kA AIRIX Electron Beam,” Proceedings PAC 2001, Chicago, 3481 (2001)

${ }^{8}$ A.C. Paul, "ETAII 20 Cell Emittance Measurement,” unpublished note dated 12 May 1997 (to be submitted as an UCLR)

${ }^{9}$ A.C. Paul, R. Richarson, and J. Weir, “ETA $6 \mathrm{MeV}$ Pepperpot Emittance Measurement,” unpublished note dated 29 June 1998 (to be submitted as an UCLR)

${ }^{10}$ M.J. Burns, P.W. Allison, R.L. Carlson, J.N. Downing, D.C. Moir, and R.P. Shurter, "Status of the Dual -Axis

Radiographic Hydrotest Facility,” Proc. of the XVIII Intl. Linear Accel. Conf., Geneva, Switzerland, 1996

${ }^{11}$ G.P. Le Sage, "Time-Resolved Emittance Characterization of an Induction LINAC Beam Using Optical Transition

Radiation,” UCRL-ID-153254, 5 Nov 2002 and unpublished measurements by J.S. Jacob performed at

${ }^{12}$ W.E. Nexsen, R.D. Scarpetti, and J. Zentler, "Reconstruction of FXR Beam Conditions,” Proceedings 2001 IEEE Particle Accelerator Conference, 2383 (2001)

${ }^{13}$ Unpublished measurement by G.A. Westenskow, performed on the FXR injector September 2001

${ }^{14}$ W.B. Herrmannsfeldt, "Electron Trajectory Program," Stanford Linear Accelerator Center report 166 (September 1973) and “An Electron Optics and Gun Design Program,” Stanford Linear Accelerator Center report 331 (October 1988)

${ }^{15}$ TriComp is a series of two-dimensional and three-dimensional, cylindrical geometry, finite-element software for electromagnetic solutions including charged particle transport. The software is created and marketed by Field Precision, PO Box 13595, Albuquerque, NM 87192 (www.fieldp.com). TriComp software used in this study included Mesh (mesh generator), ESTAT (electrostatic solver), BSTAT (magnetostatic solver), and TRAK (charged particle optics).

${ }^{16}$ K. Halbach, “A Program for Inversion of System Analysis and Its Application to the Design of Magnets,” Lawrence Livermore National Laboratory report UCRL-17436 (1967), The Poisson group of codes is maintained and distributed, without charge, by the Los Alamos Accelerator Code Group (laacg1.lanl.gov/laacg/componl.html)

${ }^{17}$ AMAZE is a series of three-dimensional, finite-element software for electromagnetic solutions including charged particle transport. The software is created and marketed by Field Precision, PO Box 13595, Albuquerque, NM 87192 (www.fieldp.com). AMAZE software used in this study included MetaMesh (mesh generator), HiPhi (electrostatic solver), and OmniTrak (charged particle optics).

${ }^{18}$ Lsp is a three-dimensional, electrodynamic, PIC code for large scale plasma simulations. The code is marketed by the Advanced Technologies Division, Mission Research Corporation, Albuquerque, NM (www.mrcabq.com).

${ }^{19}$ A.C. Paul, “TRANSPORT, an Ion Optic Program, LBL Version,” Lawrence Berkeley Laboratory report LBL-2697 (1975)

20 J.-L. Vay and W. Fawley, "Amber User's Manual," Lawrence Berkeley National Laboratory report LBNL/PUB3160 (2000)

${ }^{21}$ A.C. Paul, "Flash X-Ray Injector and Accelerator Study,” to be submitted as an FXR Note and UCLR

${ }^{22}$ T.L. Houck, etc, FXR Note 2004-May-001

${ }^{23}$ R.B. Miller, “Mechanism of Explosive Electron Emission for Dielectric Fiber (Velvet) Cathodes,” J. Appl. Phys., 84, No. 7, 3880 (1998).

${ }^{24}$ Nexsen, ibid.

${ }^{25}$ F.Hartemann, et al., “Temporal Evolution of Beam Emittance and Cathode Plasma Uniformity from a Field Emission Diode,” Proceedings of the IEEE Particle Accelerator Conference, Washington D.C., March 16-19, 1987, pp. 385-387.

${ }^{26}$ A.C. Paul, "Flash X-Ray Injector and Accelerator Study," ibid.

${ }^{27}$ Mike Ong, Paul Wargo, and Jan Zentler, “Time-resolved Cathode-velvet Light Emissions,” unpublished presentation from 3 May 2004, data taken 2 April 2004.

${ }^{28}$ F.Hartemann, et al., "Temporal Evolution of Beam Emittance and Cathode Plasma Uniformity from a Field Emission Diode,” Proceedings of the IEEE Particle Accelerator Conference, Washington D.C., March 16-19, 1987, pp. 385-387.

${ }^{29}$ A.E. Blaugrund, "Measurements of electron emission uniformity from cathodes in high-voltage electron beam diodes,” Appl. Pjys. Lett., 83, No. 6, 1264 (2003)

${ }^{30}$ Y.M. Saveliev, W.S. Sibbet, and D.M. Parkes, "Current conduction and plasma distribution on dielectric (velvet) explosive emission cathodes,” J. Appl. Phys., 94, No. 12, 7416 (2003)

${ }^{31}$ E.P. Lee and R.K. Cooper, ibid. pp. 88 and 94 